Article

\title{
Large Eddy Simulation with Energy-Conserving Schemes and the Smagorinsky Model: A Note on Accuracy and Computational Efficiency ${ }^{\dagger}$
}

\author{
Dhruv Mehta ${ }^{1,2, *, \ddagger}$, Ye Zhang ${ }^{2,3, *}$, Alexander van Zuijlen ${ }^{2}$ and Hester Bij1 ${ }^{2}$ \\ 1 Energy Research Centre of The Netherlands (now TNO), 1755 LE Petten, The Netherlands \\ 2 Aerodynamics, Wind Energy and Propulsion, Delft University of Technology (TU Delft), 2629 HS Delft, \\ The Netherlands; a.h.vanzuijlen@tudelft.nl (A.v.Z.); h.bijl@tudelft.nl (H.B.) \\ 3 School of Energy and Power Engineering, Dalian University of Technology, Dalian 116024, China \\ * Correspondence: d.mehta@tudelft.nl (D.M.); ye.zhang@dlut.edu.cn (Y.Z.) \\ + This document is a collaborative effort. The research was organised and funded through ECN (now TNO) \\ and supported by TU Delft. \\ $\ddagger$ Correspondence address: ECN TU Delft, 5.19 Kluyverweg 1, 2629 HS Delft, The Netherlands.
}

Received: 30 November 2018; Accepted: 27 December 2018; Published: 31 December 2018

check for updates

\begin{abstract}
Despite advances in turbulence modelling, the Smagorinsky model remains a popular choice for large eddy simulation (LES) due to its simplicity and ease of use. The dissipation in turbulence energy that the model introduces, is proportional to the Smagorinsky constant, of which many different values have been proposed. These values have been derived for certain simulated test-cases while using a specific set of numerical schemes, to obtain the correct dissipation in energy simply because an incorrect value of the Smagorinsky constant would lead to an incorrect dissipation. However, it is important to bear in mind that numerical codes may suffer from numerical or artificial dissipation, which occurs spuriously through a combination of spatio-temporal and iterative errors. The latter can be controlled through more iterations, the former however, depends on the grid resolution and the time step. Recent research suggests that a complete energy-conserving (EC) spatio-temporal discretisation guarantees zero numerical dissipation for any grid resolution and time step. Therefore, using an EC scheme would ensure that dissipation occurs primarily through the Smagorinsky model (and errors in its implementation) than through the discretisation of the Navier-Stokes (NS) equations. To evaluate the efficacy of these schemes for engineering applications, the article first discusses the use of an EC temporal discretisation as regards to accuracy and computational effort, to ascertain whether EC time advancement is advantageous or not. It was noticed that a simple non-EC explicit method with a smaller time step not only reduces the numerical dissipation to an acceptable level but is computationally cheaper than an implicit-EC scheme for wide range of time steps. Secondly, in terms of spatial discretisation on uniform grids (popular in LES), a simple central-difference scheme is as accurate as an EC spatial discretisation. Finally, following the removal of numerical dissipation with any of the methods mentioned above, one is able to choose a Smagorinsky constant that is nearly independent of the grid resolution (within realistic bounds, for OpenFOAM and an in-house code). This article provides impetus to the efficient use of the Smagorinsky model for LES in fields such as wind farm aerodynamics and atmospheric simulations, instead of more comprehensive and computationally demanding turbulence models.
\end{abstract}

Keywords: large eddy simulation; energy-conserving schemes; isotropic homogeneous turbulence; Runge-Kutta methods; Smagorinsky model 


\section{Introduction}

Large eddy simulation (LES) is a fine alternative for simulating high Reynolds number flows as compared to the computationally demanding direct numerical simulation (DNS). LES is tractable because it only resolves the large, energy-containing scales of a flow, while modelling the smaller and more numerous dissipative scales mathematically with a subgrid scale (SGS) model. Given the numerical complexity of this process, care must be taken in terms of detecting and handling errors stemming from spatial discretisation and the SGS model. A first insight into these errors was provided by [1], who demonstrated that the errors from spatial discretisation and turbulence modelling are equally consequential; which were later discovered to be capable of cancelling each other in certain cases, leading to a desirable result [2-4]. It was also noted that approaches designed to suppress the two sources of errors simultaneously, may not necessarily lead to a low overall error. References $[4,5]$ illustrated how the same SGS model could produce different results under the influence of different spatial discretisation schemes. The former also commented on the dependence of the Smagorinsky constant on the grid resolution [6]. Further, the accuracy of LES can also be affected by time-integration, although this can be corrected easily by reducing the time step or by using higher-order time-integration schemes.

Most of the quoted studies used a finite volume method (FVM) for solving the Navier-Stokes (NS) equations. These were combinations of 2 nd and 4 th order discretisation of the convective and diffusive terms of the NS equations, advanced in time with a 2nd order explicit Runge-Kutta scheme. Further, they commented mostly on the effect of spatial discretisation and the resulting numerical dissipation on the overall accuracy of LES. As an adjunct to these studies, this article discusses the use of energy-conserving (EC) spatio-temporal discretisation schemes for FVMs. EC schemes are designed to conserve the kinetic energy (KE) of an inviscid flow in the absence of boundaries and body forces. As a result, for a viscous flow, EC schemes ensure that the loss of KE is brought about only through the action of molecular viscosity, which is an invariant property of incompressible flows [7]. An EC spatial discretisation precludes numerical dissipation that may increase (or even reduce) the dissipation of the turbulence kinetic energy (TKE). However, concomitantly, an implicit EC time integration scheme is necessary to ensure that the conservation of KE remains enforced with time-stepping because numerical errors through time integration could also bring about numerical dissipation.

In keeping with above, this article first addresses whether an implicit EC time integration scheme is crucial for averting numerical dissipation or could one use an explicit non-EC scheme and yet reduce the numerical dissipation to a value that the resulting solution is accurate? This is solely because implicit EC schemes are computationally demanding and numerically complex to implement, as a result of which, they may not be most suited for engineering applications that concern the research described in this article, namely, the simulation of the atmospheric boundary layer (ABL) and wind farm aerodynamics.

Secondly, the article compares an EC spatial discretisation with the simple central difference scheme. An EC spatial scheme is based on arithmetic means but on a staggered grid. On the other hand, a central difference scheme is based on arithmetic means on a collocated grid. As a result, both schemes do not have a dissipative error per se. This article considers only Cartesian grids on which the theoretical advantages of EC schemes have been established. but have a non-zero dispersive error. This article assesses whether there is a difference between the EC and central difference approaches (on their respective grids) in terms of how they affect the outcome in LES.

The tests described in this article are simulated using the simple central difference scheme on collocated grids available in the open source field operation and manipulation (OpenFOAM) toolbox [8] and the EC schemes within the Energy-Conserving Navier-Stokes (ECNS) code [9] owned by the Energy research Centre of the Netherlands (ECN, now TNO).

To avoid confusion, the term dissipation-free instead of energy-conserving will be used to indicate the property of conservation of energy obeyed by at least, the EC scheme and must be obeyed by any dissipation-free scheme in general. Therefore, the abbreviation EC, henceforth strictly refers to the 
numerical scheme offered within the ECNS and not the property of being dissipation-free. Further, the term EC-discretisation refers to discretisation of the convective operator in the NS equations.

Table 1 lists the features of the ECNS and OpenFOAM in terms of spatial discretisation. The only aspect in which these two FVM-based codes differ, is their formulation on non-uniform grids. The EC scheme remains dissipation-free as it uses the arithmetic mean to interpolate and average the velocity field across cell boundaries [7]. Whereas, the central difference scheme in OpenFOAM switches to linear interpolation on non-uniform grids. This article assesses if this difference makes one scheme more favourable than the other and under what circumstances. Finally, the article discusses whether the absence of numerical dissipation promotes the use of the simple Smagorinsky model for the LES of turbulent flows with FVMs.

Table 1. Salient features of the two dissipation-free spatial discretisation methods studied in this article.

\begin{tabular}{ccc}
\hline Scheme & ECNS & OpenFOAM \\
\hline Grid & Staggered & Collocated \\
Interpolation & Arithmetic mean & Linear \\
Differencing & Central & Central \\
\hline
\end{tabular}

It is important to emphasise that this article is aimed solely at assessing how effectively the Smagorinsky model can be adapted to simulate flows with the EC schemes, towards ascertaining if such an approach could indeed find its way into practical applications in engineering.

\section{LES and the Smagorinsky Model}

The first step in LES involves modifying the NS equations to represent only the large scales, specifically, the scales greater than a cut-off length, $\Delta[10,11]$. This operation that is formally defined as filtering, when applied to the incompressible form of the NS equations leads to,

$$
\begin{gathered}
\frac{\partial \widetilde{u}_{i}}{\partial x_{i}}=0, \\
\frac{\partial \widetilde{u}_{i}}{\partial t}+\frac{\partial \widetilde{u_{i} u_{j}}}{\partial x_{j}}=-\frac{\partial \widetilde{p}}{\partial x_{i}}+\frac{\partial}{\partial x_{j}}\left(v\left(\frac{\partial \widetilde{u}_{i}}{\partial x_{j}}+\frac{\partial \widetilde{u}_{j}}{\partial x_{i}}\right)\right),
\end{gathered}
$$

where the pressure $p$ has been divided by the constant density of the fluid and $v$ is the kinematic viscosity.

As opposed to the standard representation of the component of velocity in the $i$-th direction as $u_{i}, \widetilde{u}_{i}$ represents the velocity field of the scales greater than $\Delta$. In practice, $\Delta$ is generally equal to the grid size and hence, all scales that are smaller than $\Delta$ are called the subgrid scales. Next, the filtered convective term is further simplified using Leonard's decomposition [12] or,

$$
\tau_{i j}=\widetilde{u_{i} u_{j}}-\widetilde{u_{i}} \widetilde{u}_{j}
$$

which leads to,

$$
\frac{\partial \widetilde{u}_{i}}{\partial t}+\frac{\partial \widetilde{u}_{i} \widetilde{u}_{j}}{\partial x_{j}}=-\frac{\partial \widetilde{p}}{\partial x_{i}}+\frac{\partial}{\partial x_{j}}\left(v\left(\frac{\partial \widetilde{u}_{i}}{\partial x_{j}}+\frac{\partial \widetilde{u}_{j}}{\partial x_{i}}\right)\right)-\frac{\partial \tau_{i j}}{\partial x_{j}}
$$

where $\tau_{i j}$ is the SGS tensor that represents the interaction between the subgrid scales and the large resolved scales. The SGS tensor is generally represented by SGS models that are semi-empirical. For more details, the readers are referred to two important reviews on this subject by $[13,14]$. The Smagorinsky model that is used in this article is based on expressing $\tau_{i j}$ in terms of the resolved velocity field and eddy viscosity as [6],

$$
\tau_{i j}-\frac{1}{3} \delta_{i j} \tau_{k k}=-2 v_{T} \widetilde{S}_{i j}
$$


where $\widetilde{S}_{i j}$ is tensor of the resolved strain rate or the strain rate of the resolved velocity field. $v_{T}$ is the eddy viscosity, which in terms of the resolved velocity field is defined as,

$$
v_{T}=\left(C_{S} \widetilde{\Delta}\right)^{2}\left(2\left|\widetilde{S}_{i j}\right|^{2}\right)^{\frac{1}{2}}
$$

where $C_{S}$ is the flow-specific Smagorinsky constant. The values reported for $C_{S}$ range from 0.1 to 0.23 , of which most have been deduced from experimental or DNS data (see [15]).

In practice, LES codes report different values of $C_{S}$ purely based on the study of decaying turbulence alone. Reference [16] proposed a value of 0.17 . Reference [17] obtained a value of 0.144 with the EllipSys3D LES code [18,19], whereas [5] used 0.15. This article will also report different values of $C_{S}$ for the ECNS and OpenFOAM.

These different values stem from different discretisation schemes and their errors that are always present unless the grid is very fine and the time step very small, all of which affect how the Smagorinsky model must be tuned to obtain the correct overall dissipation. It is important to note that the Smagorinsky model does not involve explicit filtering of the velocity field. The grid itself acts as the filter that only resolves all scales bigger than the grid size. Further, $\widetilde{\Delta}$ is the cut-off scale in space that in practice should be set equal to $\Delta_{g}$, the overall grid size, which is $\Delta_{g}=\sqrt[3]{\prod_{i=1}^{3} \Delta_{i}}$, where $\Delta_{i}$ is the average grid size along the $i$-th direction. One may choose a larger value of $\widetilde{\Delta}$ in which case, the Smagorinsky model only parameterises the scales smaller than $\widetilde{\Delta}$ that must then be referred to as the subfilter scales (SFS). In this article, $\Delta_{i}$ is fixed as the overall grid size. More details on the relationship between subgrid and subfilter scales can be found in $[3,20]$.

\section{Dissipation in a Numerical Scheme}

Within the discrete NS-LES system, the errors that arise can be classified as those from the regular operators of the discrete NS equations or those from the SGS model. The former comprises errors from the discretisation of the convective and diffusive terms, while the latter comprises the modelling error due to the nature of the SGS model and the discretisation error through the implementation of the SGS model in the NS solver.

One can roughly express the overall dissipation in an LES scheme as,

$$
\mathcal{P}=\varepsilon_{C}(\Delta x)+v\left(\mathcal{D}+\varepsilon_{D}(\Delta x)\right)+\mathcal{S}(\tau)+\varepsilon_{S}(\Delta x)+\varepsilon_{T}(\Delta t) .
$$

$\mathcal{D}$ and $\mathcal{S}$ represent the diffusive (physical diffusion) and the SGS operator, respectively. $\varepsilon_{C}$ and $\varepsilon_{D}$ are the discretisation errors (dissipative) accompanying the convective and diffusive operators, respectively. $\varepsilon_{D}$ (dissipative) arises from the approximation used to estimate the physical diffusion through molecular viscosity. Dissipation through the SGS model is represented by a function of the model $\tau$, which can be tuned (see Section 2) and the spatial discretisation error in the model's implementation $\varepsilon_{S}$, which is a function of the grid resolution $\Delta x$. The pressure term also leads to numerical dissipation but not with an EC scheme, this dissipation will be ignored for now.

$\varepsilon_{T}$ is the dissipation through time integration, which is a function of the time step and must approach zero with reducing time step. It is also known from [21] that numerical dissipation through time integration can alter how the flow evolves and hence, must also be accounted for in the above equation.

Physical dissipation or $\mathcal{P}$, pertains to the dissipation that one would try to achieve with a numerical method, which should equal what the flow would experience in nature. In a high Reynolds number flow, the molecular diffusion is very small as compared to the turbulent diffusion and so is the error from the discrete diffusive operator, both of which can be neglected. In LES of wind farms and the atmospheric boundary layer, the diffusive term is set to zero. However, the diffusive term has not been neglected for the low Reynolds number tests reported in this article. Assuming that the error 
from time-integration is reduced with a small time step or an EC time integration scheme, Equation (7) then reads,

$$
\mathcal{P}=\varepsilon_{C}(\Delta x)+\mathcal{S}(\tau)+\varepsilon_{S}(\Delta x)
$$

In a dissipation-free spatial scheme, the error from the convective operator is predominantly dispersive (see [21]). For a high Reynolds number flow, the convective term is large as compared to the term representing molecular diffusion. It is therefore likely that numerical dissipation from the discrete convective term could be comparable the term representing the discrete molecular diffusion. In doing so, the numerical dissipation through $\varepsilon_{C}$ can influence the flow to a great extent and should be removed. As a consequence with an LES code based on a dissipation-free scheme, one would expect Equation (8) to read,

$$
\mathcal{P}_{E C} \approx \mathcal{S}(\tau)+\varepsilon_{S}(\Delta x) .
$$

Whereas, with a non-EC scheme one obtains,

$$
\mathcal{P}_{\text {Non }-E C} \approx \varepsilon_{C}(\Delta x)+\mathcal{S}(\tau)+\varepsilon_{S}(\Delta x) .
$$

Next, the dissipative errors from the implementation of the SGS model and $\varepsilon_{S}(\Delta x)$ are combined with the dissipation arising from the model itself i.e., $\mathcal{S}(\tau)$, to rewrite the above equations as,

$$
\begin{gathered}
\mathcal{P}_{E C} \approx \mathcal{S}(\tau, \Delta x), \\
\mathcal{P}_{\text {Non-EC }} \approx \varepsilon_{C}(\Delta x)+\mathcal{S}(\tau, \Delta x) .
\end{gathered}
$$

It is hard to assess the contribution of $\Delta x$ within $\mathcal{S}(\tau, \Delta x)$ but it is nonetheless apparent that the contribution of $\Delta x$ as a whole to $\mathcal{P}$, reduces with the removal of $\varepsilon_{C}(\Delta x)$. Therefore, it is fair to hypothesise that with a non-dissipative scheme (EC or pseudo-spectral), the dissipation is unaffected by the grid resolution to a greater extent that what would be the case with a scheme that has inherent numerical dissipation.

This property may help tune the SGS model more easily as the grid resolution has a relatively minor role to play, when $\varepsilon_{C}(\Delta x)$ is absent or very small compared with $\mathcal{S}(\tau)$ (if some numerical dissipation is present). In fact, the property of inherent numerical dissipation in terms of $\varepsilon_{C}(\Delta x)$ is put to good use by Implicit LES methods wherein, the dissipation introduced by the discretisation replaces the SGS model (see [22]).

Although one cannot completely rule out the dependence of the SGS model on grid resolution (or $\varepsilon_{S}(\Delta x)$ ), evidence from literature suggests that within a reasonable range of grid resolutions, the dependence is moderate enough for practical applications such as the simulation of high Reynolds number flows within the ABL (confirmed for the Smagorinsky model in [23]). It has been observed that at poor grid resolutions, the optimal Smagorinsky constant is quite dependent on the grid resolution. However, once the grid resolution is sufficient to promote adequate resolution of the large scales relevant for high Reynolds number flows, the dependence on increasing grid resolution is relatively weaker.

\section{ECNS and OpenFOAM}

This section provides information on the two codes considered in this article, ECNS and OpenFOAM; and the relevant spatial discretisation and time integration schemes.

\subsection{ECNS}

The ECNS offers 2nd order spatial discretisation on a staggered and Cartesian grid. For time integration, one can choose from the classical explicit 4th order but non-EC Runge-Kutta scheme (Ex4) and the implicit 2nd and 4th order EC Runge-Kutta schemes based on the Gauss quadrature, henceforth 
referred to as the $\operatorname{Im} 2$ and $\operatorname{Im} 4$. These time integration schemes are summarised in Appendix A; one can find the details in [21].

\subsection{OpenFOAM}

OpenFOAM (http:/ / www.openfoam.com) is an open-source simulation package that permits the user to customise an NS solver using a combination of spatial-temporal schemes and a turbulence model. It uses a finite volume approach like the ECNS.

Amongst the host of schemes offered, the 2nd order central difference-based Gauss Linear scheme has inherently zero numerical dissipation but a non-zero dispersive error [24]. In addition, the inherently dissipative quadratic upwind interpolation scheme for convective kinematics (3rd order, QUICK) and the linear upwind (2nd order, LU) schemes are also considered. As regards to Table 1, QUICK uses a collocated uniform grid but with upwind differencing and interpolations defined by the QUICK scheme itself. For time integration, the Crank-Nicolson 2nd order implicit forward time stepping is used with a very small time step to avert numerical dissipation through time integration [24].

It is important to mention that improper coupling of pressure and velocity within a solver can affect the KE. Appendix A provides the relevant details. For simplicity, the spatial discretisation approach with the EC scheme on staggered grids is directly referred to as ECNS and the central difference scheme on collocated grids as OpenFOAM.

\section{Tests}

The first objective is to determine what time integration schemes require the least amount of computational effort while guaranteeing that the numerical dissipation through time integration does not affect the flow.

Secondly, this article establishes qualitatively whether Equations (11) and (12) hold for the spatial discretisation approaches mentioned in Table 1. Next, the consequences that result should Equations (11) and (12) not hold, would be discussed followed by an assessment of whether the differences between a central difference scheme on a collocated grid and an EC scheme on a staggered grid, lead to marked differences the results so-obtained.

\subsection{Grid-Generated Turbulence}

The test case simulated in this article is the recognised experimental campaign by [25] (CBC), who used a grid to generate an isotropic homogeneous turbulent field in a wind tunnel. The experiments provided details on the energy spectrum and the statistical properties of the turbulent flow at three instances of time, as the flow moves through a wind tunnel at a near constant average velocity, $U_{o}=10 \mathrm{~ms}^{-1}$. This case was chosen as it has been widely used for the validation of LES codes and also for the tuning of the Smagorinsky model [17].

One must bear in mind that the flow's Reynolds number is low, around 34,000 based on the average velocity and the size of the grid used to create turbulence $\left(M_{g}=5.08 \mathrm{~cm}\right)$ and that the flow is not truly isotropic with an isotropy ratio of 0.95 (the ratio of the standard deviation in streamwise velocity to that in transverse velocity). Hence, the TKE is higher in the transverse direction than in the streamwise direction.

Although the slight anisotropy can be overlooked, the low Reynolds number requires attention given that the fundamentals of LES, by definition, consider a high Reynolds number flow with an extended inertial range [10]. Further, the experimental energy spectra in the inertial range deviate a little from the $-5 / 3$ Kolmogorovspectrum that is observed in turbulent flows [25-27] (see Figure 1). These cannot be verified even through DNS because of the wide range of length and time scales in the flow, for which the current computational resources are insufficient.

Nevertheless, this article provides an assessment of how closely the simulations can replicate the spectra from the largest to the smallest scales on the grid, which indirectly should also estimate the correct decay of TKE with time. It is fair to mention that the intention is not to validate the ECNS or 
perform a follow-up study of the experiments by [25]. Other relevant test cases from literature were also considered; Appendix B provides the details. Apart the cases mentioned in Appendix B, the ECNS was also tested against the roll-up of a shear layer, the simulation of the ABL and the experimental analysis of a single wind turbine in turbulent flow, while being compared against a range of LES codes available in literature. More details on these simulations can be found in [28,29].

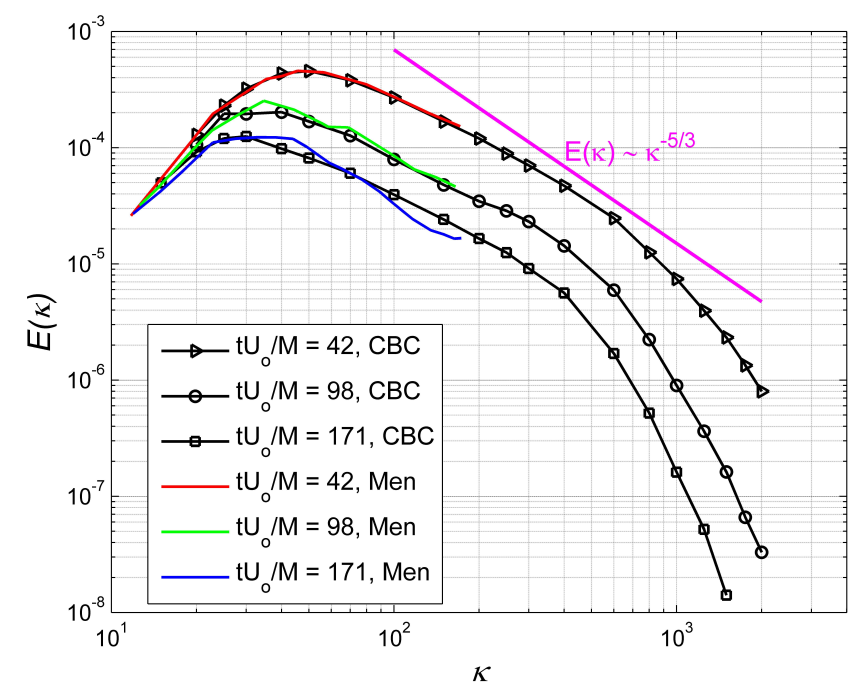

Figure 1. A comparison of the energy spectra obtained by [25] and those using a $32^{3}$ grid with the dynamic Smagorinsky model with Lagrangian-averaging by [26] (Men in the graph). $\kappa$ is the wave number related to an eddy's length scale and $E(\kappa)$ is the eddy's energy.

\subsection{Effect of Time Step and Time Integration}

One notices three experimentally obtained spectra in Figure 1 (CBC). These correspond to three different locations in a wind tunnel at which the flow was analysed. The codes are used to simulate the flow between Stages I and III, referred to as $U_{0} t / M_{g}=42$ and 171, respectively, in [25] with an intermediate stage, $U_{0} t / M_{g}=98$ or Stage II, where $t$ is the time. These numbers refer to distances downstream of the grid that generates the turbulence. The generation of the initial turbulent velocity field using the experimental data is explained in Appendix $C$.

The CBC test case is simulated on $32^{3}, 48^{3}$ and $64^{3}$ uniform 3D grids with average grid sizes $\left(\Delta_{g}=\sqrt[3]{\prod_{i=1}^{3} \Delta_{i}}\right)$ equalling $1.72 \mathrm{~cm}, 1.14 \mathrm{~cm}$ and $0.86 \mathrm{~cm}$, respectively. These sizes are small as compared to the longitudinal length scale of $2.4 \mathrm{~cm}$ but larger than the Taylor micro scale of $0.48 \mathrm{~cm}$ at Stage I, which places the cut-off filter (grid-based) somewhere in the inertial range, as the case should be. Therefore, a $16^{3}$ grid would be quite coarse. In certain cases, $80^{3}$ and $96^{3}$ grids are also used. With regards to the time-stepping, the arguments are centred on a time step of $\Delta t=0.0001 \mathrm{~s}$. This time step was chosen because it was used as reference by [17] to ensure that the Courant number was below 0.1 for a second order implicit time integration scheme, while tuning an LES code with the CBC case for two different grid resolutions.

Further, the time integration schemes are operated with a range of pre-determined time steps. When a time integration scheme is used with $\Delta t=0.001 \mathrm{~s}$, it will be quoted by its name alone, i.e., as Ex4 or Im2. For other time steps, an extra letter is added at the end of the scheme's name, for example, Ex4Q. The time steps and their corresponding letters are summarised in Table 2 with Ex4 to serve as an example.

For most simulations with the Smagorinsky model, the $C_{S}$ is set to 0.1 [30], which will later be determined more accurately for the ECNS. As an output, the energy spectrum, the rate of decay of TKE and the computational time are monitored. Based on how accurately the spectra and decay of 
TKE are predicted and with what computational effort, this article will outline which time integration scheme is most appropriate for the ECNS. Using the $32^{3}$ grid, the CBC experiment is simulated with Ex4, Im 2 and Im4 (the three schemes available within the ECNS), in combination with different time steps, the acronyms for which are defined in Table 2 . The reason behind choosing the $32^{3}$ grid apart from being computationally efficient, is that the energy spectra from $\mathrm{CBC}$ suggest that the inertial range is not that wide; contrary to what is expected of a high Reynolds number flow. Although LES can resolve the large energy-containing range and a part of the inertial range but one must avoid resolving the lower inertial range that is comparable with the Kolmogorov scales, most of which are represented by the SGS model. Ideally, the cut-off scale should be in the inertial range such that it is comparably smaller (say a factor of 10 to 100) than the large scales but larger by a similar factor with respect to the Kolmogorov scales (see [10] for a discussion).

Table 2. A summary of the time steps used.

\begin{tabular}{cc}
\hline Notation & Time Step \\
\hline Ex4Q & $0.004 \mathrm{~s}$ \\
Ex4D & $0.002 \mathrm{~s}$ \\
Ex4 & $0.001 \mathrm{~s}$ \\
Ex4h & $0.0005 \mathrm{~s}$ \\
Ex4q & $0.00025 \mathrm{~s}$ \\
Ex4te & $0.0001 \mathrm{~s}$ \\
\hline
\end{tabular}

\subsubsection{Stability}

The stability of the Ex4 is conditionally determined by the Courant Friedrichs Lewy (CFL) criterion for explicit time integration schemes. Therefore, at time steps larger than $0.004 \mathrm{~s}$, the simulation was unstable with a Courant number greater than $\sim 2.4$. Reducing the time step to obtain a Courant number below 1 , for example $t=0.001 \mathrm{~s}$, guarantees stability. On the other hand, owing to its unconditional stability, the $\operatorname{Im} 2$ is stable even at a time step of $0.01 \mathrm{~s}$ that is ten times the reference time step, as opposed to the Ex4 that is divergent [21].

Now to compare the three methods, one must ensure that they all are stable at the time step considered. Therefore, the Ex4 would be used only with time steps smaller than $0.002 \mathrm{~s}$.

\subsubsection{Error Analysis}

First, a reference solution is calculated using Ex4 with a very small time step of $0.00005 \mathrm{~s}$, which was sufficiently low to ensure an error below $\left(10^{-9}\right)$ through time integration, for all the time integration schemes considered here. Next, the root-mean-square of the difference between the TKE of the field obtained during a simulation and the TKE of the reference solution (interpolated in time for the relevant time step), is analysed over the duration of the simulation. The TKE is defined as,

$$
\mathrm{TKE}=\frac{1}{2}\left(\sum_{i=1}^{3} \overline{u_{i}^{\prime} u_{i}^{\prime}}\right),
$$

where $\overline{u_{i}^{\prime} u_{i}^{\prime}}$ is the standard deviation in the component of velocity along the $i$-th direction. This value is in fact, the average KE associated with the fluctuations in velocity across the range of eddies within the flow (presented as energy per unit mass). The TKE is chosen instead of the KE as the changes in KE in the $\mathrm{CBC}$ case are hard to detect due to the high average streamwise velocity of $10 \mathrm{~ms}^{-1}$ as compared to velocity fluctuations that are only $2.22 \%$ of the streamwise value (see [25] for experimental values). The error is calculated using the following definitions of $e_{2}$ and $e_{\infty}$,

$$
e_{2}=\left(\frac{1}{n} \sum_{i=1}^{n}\left|\varepsilon_{i}\right|^{2}\right)^{\frac{1}{2}}
$$




$$
e_{\infty}=\max \left|\varepsilon_{i}\right|,
$$

where $\varepsilon$ is the difference between the TKE of the reference solution and the simulated TKE at a given instance of time and $n$ is the number of such observations. Figure 2 shows the change in TKE with time as obtained with various time steps and time integration schemes. The errors are shown in Figure 3 with the computational time of the simulation. $T$ is the time needed to simulate the test case on a $32^{3}$ grid using Ex4 and $\Delta t=0.001 \mathrm{~s}$.

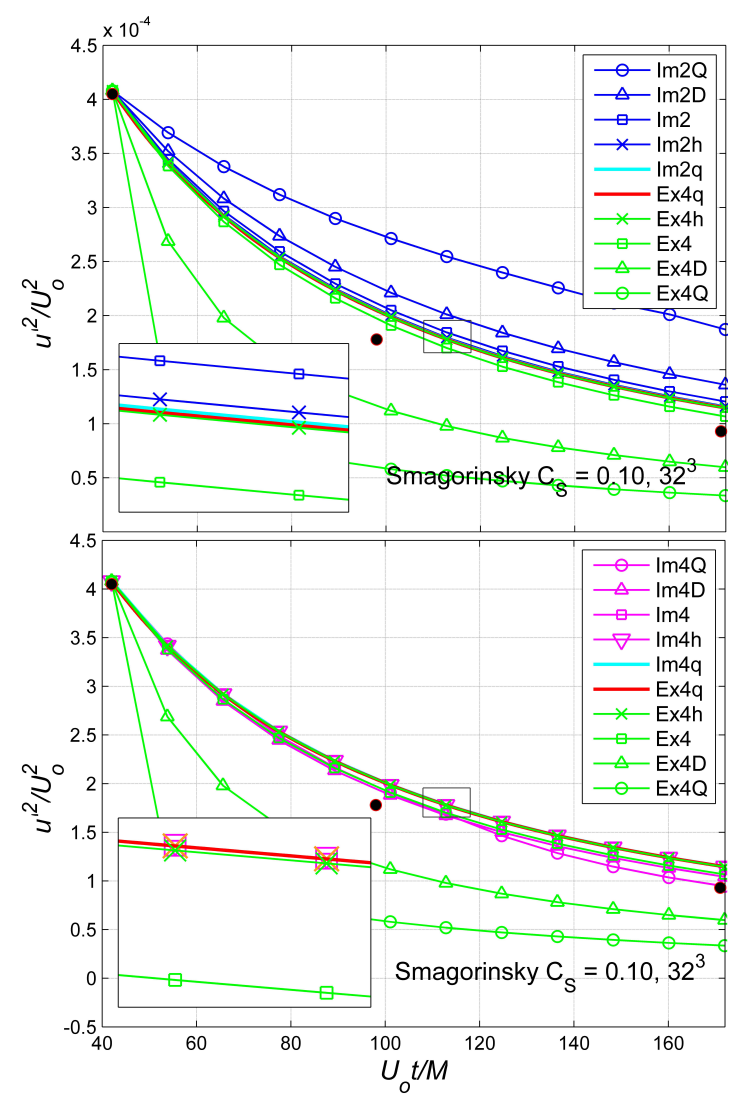

Figure 2. A comparison of the decay of TKE as predicted by the Ex4-Im2 (above) and Ex4-Im4 (below) methods with various time steps on a $32^{3}$ grid.

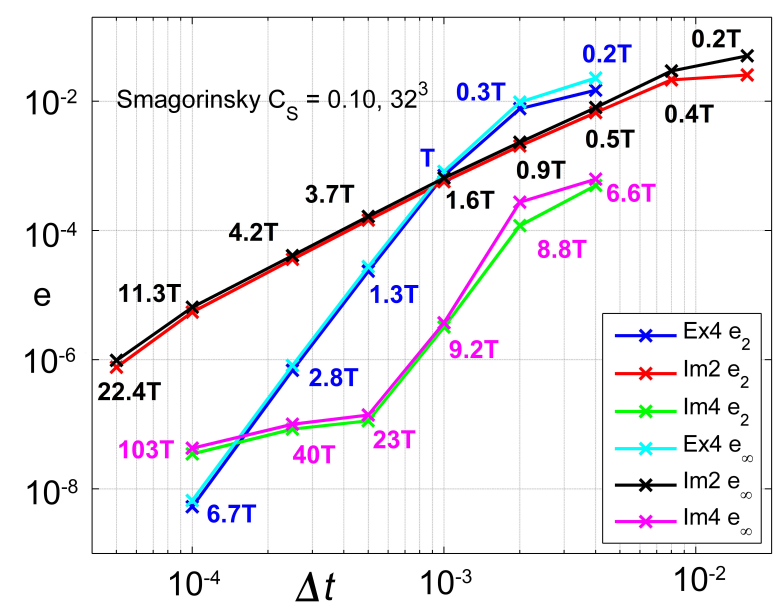

Figure 3. An error analysis based on the decay of TKE on a $32^{3}$ grid. The reference solution is obtained with Ex4 and a time step of $0.0002 \mathrm{~s}$. $T$ is the time required to obtain a solution with Ex4. 


\subsubsection{Order}

Figure 3 shows the 2nd order accuracy of the Im 2 and the 4th order accuracy of the Ex4 and Im4 (design orders).

For a time step larger than $0.001 \mathrm{~s}$, the Ex4 incurs more error as compared to the Im2. This is best explained in terms of the methods' orders of accuracy. The $\operatorname{Im} 2$ converges with second order accuracy, hence the reduction (or increase) in error with the refinement of time step should be less than what is noticed with the fourth order Ex4. Therefore, $\operatorname{Im} 2$ is more accurate at larger time steps with a lower magnitude of error. But as the time step is reduced, it is the non-EC Ex4 method that is more accurate.

Im4 leads to the lowest global error at nearly all time steps. However, at very small time steps, one observes that Im4 incurs a larger error than Ex4 and loses its 4th order accuracy. One of the reasons behind this is that the iterative method being used to solve the Im 4 system reaches its limit of accuracy as the tolerance cannot be reduced further due to computational restrictions. Further, at very large time steps $(0.004 \mathrm{~s})$, the accuracy is compromised because the time step is not sufficient to resolve the turbulent phenomena that may have a time scale that is smaller than the time step. Figure 4 presents a similar analysis on $48^{3}$ and $64^{3}$ grids. One notices slopes corresponding to the accuracy of the relevant method, independent of the grid resolution. Further, the error is larger with refined grids at a given time step, as one must ideally reduce the time step to maintain the same Courant number.

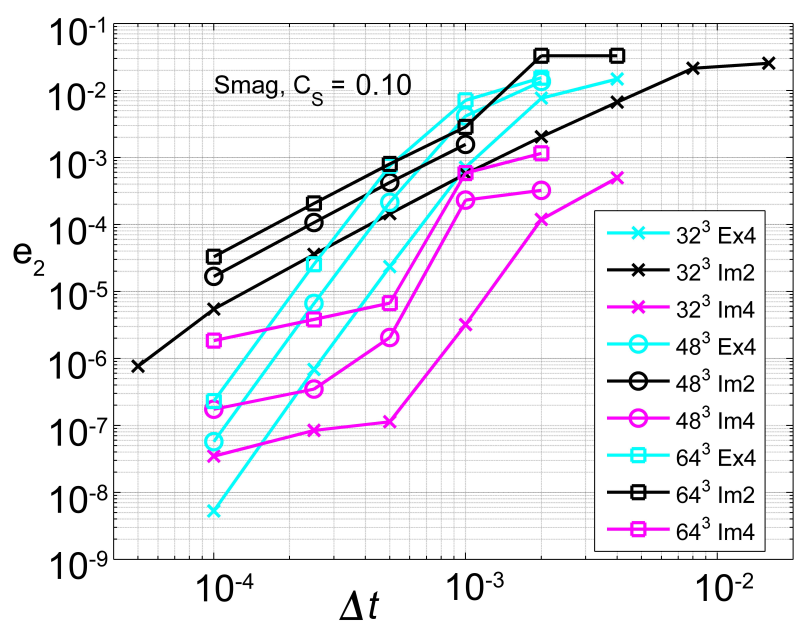

Figure 4. An error analysis based on the decay of TKE on different grids.

\subsubsection{Accuracy}

To evaluate the accuracy of the time integration schemes, one must have the correct value of $C_{S}$. Therefore, this article evaluates the effect of changing the time step and the time-integration scheme on a reference solution calculated with a fixed value of $C_{S}$. These changes are evaluated in terms of the energy spectrum, which is the distribution of the TKE across the various scales of turbulence. This will be helpful in the next section that outlines the tuning of the Smagorinsky model. If the model is tuned on the basis of the TKE, one would ensure that the total TKE is correctly predicted. But tuning with respect to the spectra will not only ensure a correct value of TKE but also its distribution within the various scales of turbulence.

In Figure 5 (compare the energy spectra at Stages II and III), one notices that a time step of $0.002 \mathrm{~s}$ with the Ex4 results in a large amount of numerical dissipation as compared to $0.001 \mathrm{~s}$, causing a marked change in the energy spectra; and the decay of TKE, as shown previously in Figure 2. The plot also presents the ratio of the energy at a given wavenumber for a certain time step, to the energy at the same wavenumber obtained with the reference solution of Ex4 with a time step of 0.00005 s; mentioned as $A_{T w}$ in the bottom-right plot within Figure 5. This helps visualise the distribution of the error in predicting the energy across wavenumbers. A value close to 1 indicates an accurate prediction, whereas values less than or greater than 1 indicate an under prediction or an over prediction, respectively. One 
notices that reducing the time step below $0.001 \mathrm{~s}$ does not lead to marked changes in the spectra $\left(A_{T w}\right.$ is nearly 1).

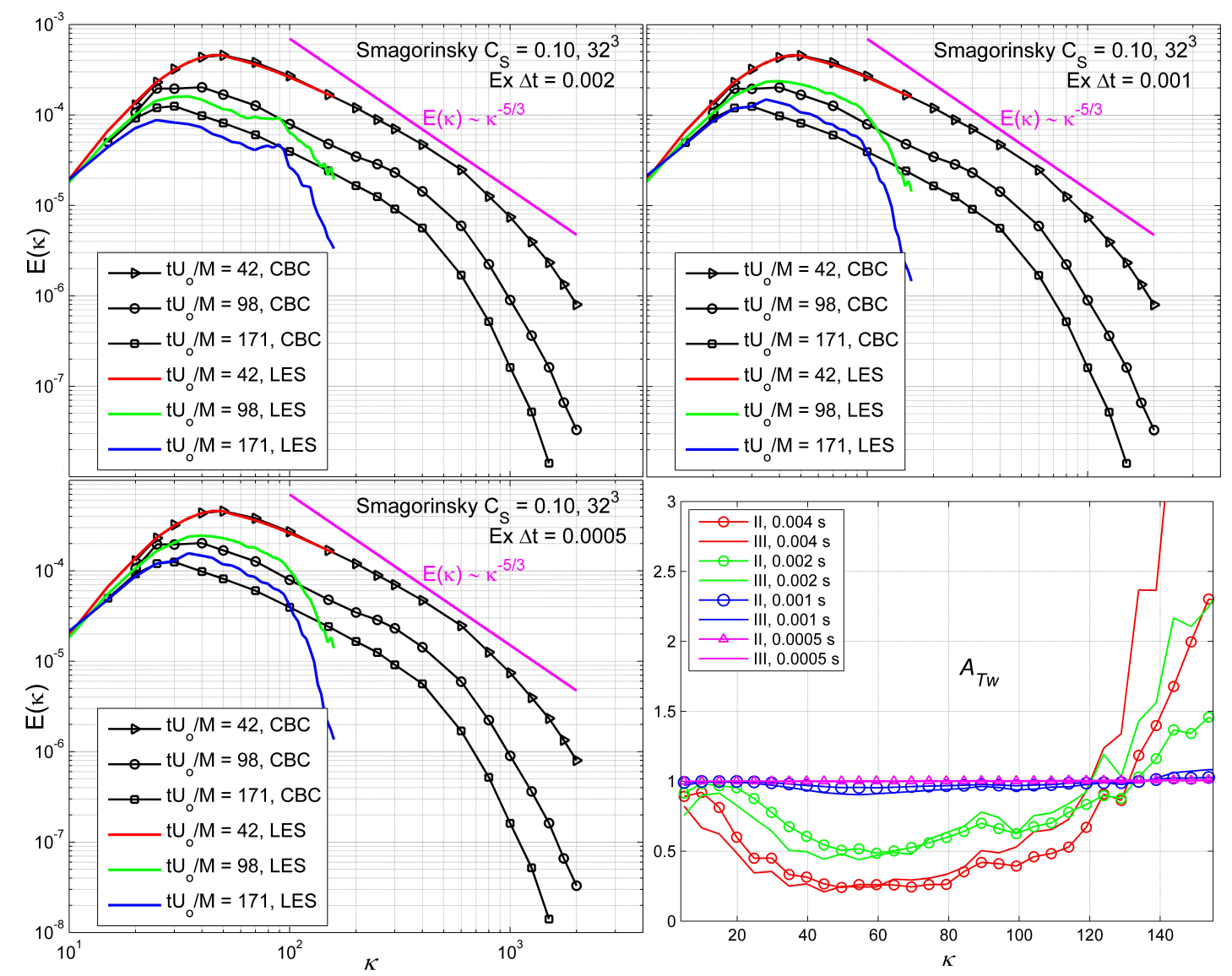

Figure 5. Energy spectra obtained using $C_{S}=0.1$ on a $32^{3}$ grid with the Ex4 and different time steps.

Comparing the error analysis (see Figure 4) with the change in TKE with time shown in Figure 2, one can establish that an error below $10^{-4}$ pertains to a solution obtained without significant influence of numerical dissipation through time integration. With the Ex4, this time step is below $0.005 \mathrm{~s}$ or a Courant number of less than 0.15 , indicating that even with a higher order method may need a small time step to avert numerical dissipation.

On the other hand, $\operatorname{Im} 2$ requires a time step of $0.0025 \mathrm{~s}$ due to its lower order of accuracy, to obtain a final error below $10^{-4}$. But the Im4 being 4th order accurate and EC by design, is accurate enough in this case at a time step of $0.002 \mathrm{~s}$ itself (in fact, even at a time step of $0.002 \mathrm{~s}$ ), as shown in Figure 6. As a result, one also notices that the spectra obtained with $\operatorname{Im} 4$ do not show much apparent variation in comparison with the spectra obtained with the reference solution of Ex4 with $0.00005 \mathrm{~s}$. The $A_{T w}$ remains nearly 1 at almost all the wavenumbers (see bottom-right plot in Figure 6).

One could conclude that Im4 is highly accurate as compared to the other two methods. However, it is important to consider the computational costs that one must reckon with while using higher-order implicit EC methods. 


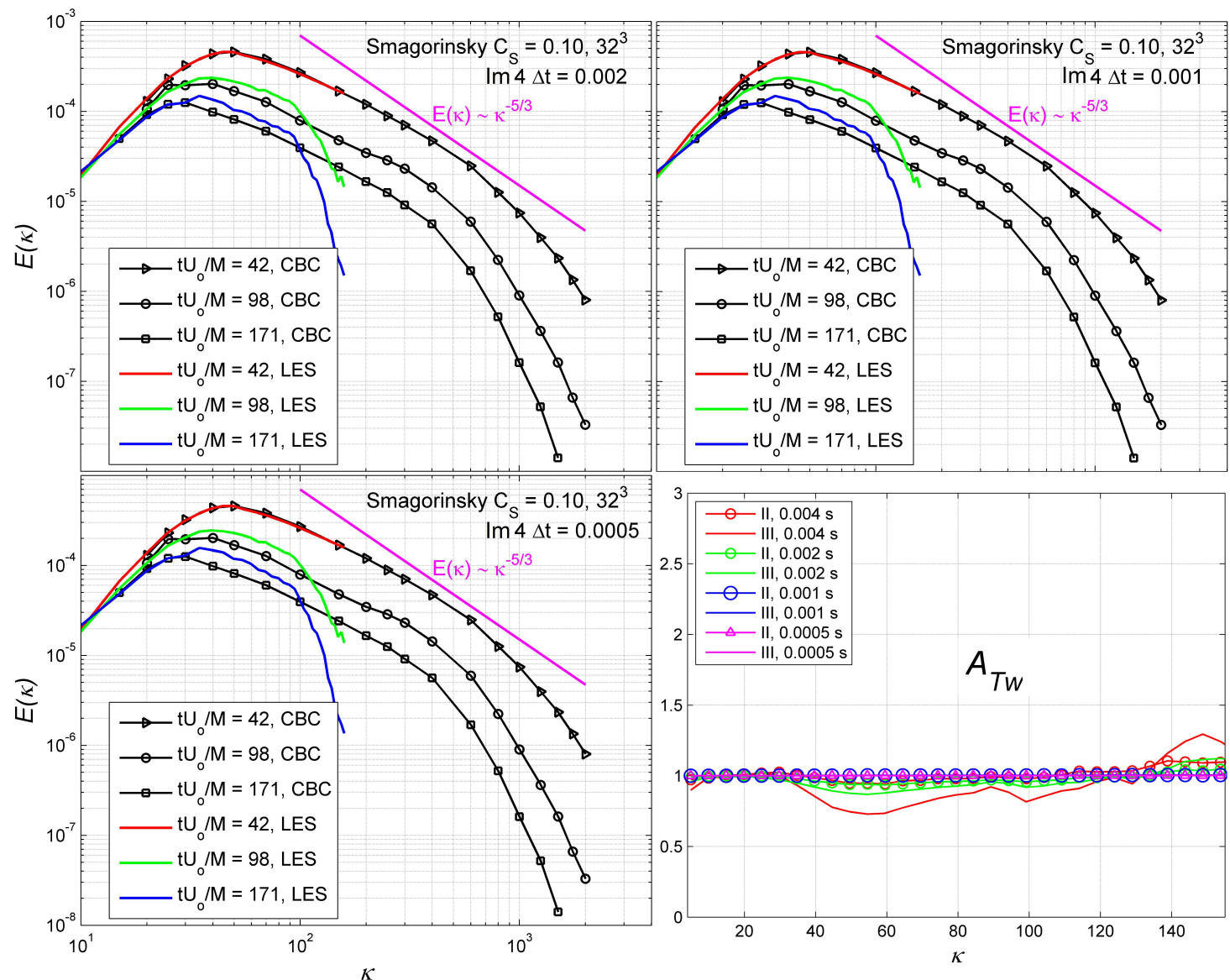

Figure 6. Energy spectra obtained using $C_{S}=0.1$ on a $32^{3}$ grid with the $\operatorname{Im} 4$ and different time steps.

\subsubsection{Efficiency}

From the preceding discussion on accuracy, it is clear that Ex4 with a time step of $0.0005 \mathrm{~s}$ on a $32^{3}$ grid, leads to an accurate solution that is independent of time step, with a computational time of 1.3T, where $T$ is the time required by Ex4 (see Figure 3). This corresponds to an error below $10^{-4}$. The computational times on finer grids show a similar trend with the various schemes and time steps. These are not discussed for succinctness.

Upon reducing the time step, the error is driven below $10^{-8}$ with Ex4 using a time step of $0.0001 \mathrm{~s}$, costing about 6.7T in computational time due to the method's high order of accuracy. On the other hand, the Im 4 permits using a larger time step of $0.002 \mathrm{~s}$ but with a very high computational time of 8.8T. This computational time is very high to the extent that $E x 4$ with a time step of $0.0001 \mathrm{~s}$ is as fast as $\operatorname{Im} 4$ with a time step of $0.004 \mathrm{~s}$.

Hence, the advantage of an implicit method permitting a larger time step, is annulled by the increased computational time required per time step. Although the ECNS is not parallelised, it is safe to believe that the ratios of computational times with the implicit methods presented in Figure 3, would at best remain the same upon parallelisation, as compared to the Ex4. An improved computational efficiency with the implicit methods would require a more optimised approach to solving the coupled system of equations (see Appendix D for a detailed discussion).

\subsection{Effect of Spatial Discretisation Schemes}

The first step to a fair comparison of the ECNS and OpenFOAM with regards to LES, is to tune the Smagorinsky model for the two codes. As per the hypothesis and Equations (11) and (12), the absence of numerical dissipation may reduce the influence of grid resolution on the overall dissipation, which in the case of the Smagorinsky model can be controlled with the Smagorinsky constant or $C_{S}$ (this 
represents $\varepsilon_{S}$ and $\mathcal{S}$ in Equations (11) and (12)). Therefore, the absence of numerical dissipation may ensure that $C_{S}$ is grid-independent to a greater extent with non-dissipative schemes as compared to a code that uses dissipative spatial discretisation.

\section{Sensitivity of the Spectra to Changes in Grid Resolution}

For the time being, assume $C_{S}=0.12$. It shall be shown later that $C_{S}=0.12$ or 0.125 is appropriate for the ECNS. This value is merely used to help the reader understand the discussion that follows. for the ECNS. Figure 7 shows the energy spectra at different grid resolutions. Further, the bottom-right plot in the Figure 7 shows the ratio of the simulated and the experimentally determined value of the energy as a function of the wavenumber; represented by the symbol $A_{C B C}$.

One notices that the predicted spectra follow the experimental curve till a certain wavenumber beyond which, the predicted spectra show a slight deviation (dampening in magnitude) followed by an abrupt and large reduction in energy just before the cut-off wavenumbers (these abrupt changes and large deviations would be called damped tails). It is observed that upon increasing the resolution beyond $48^{3}$, the abrupt dampening continues to occur but shifts to higher wavenumbers. However, the lower wavenumbers are accurately predicted and are affected to a minor extent by the increasing grid resolution. This can be seen through the plot of $A_{C B C}$ wherein the lines are clustered till a wavenumber of 100 .

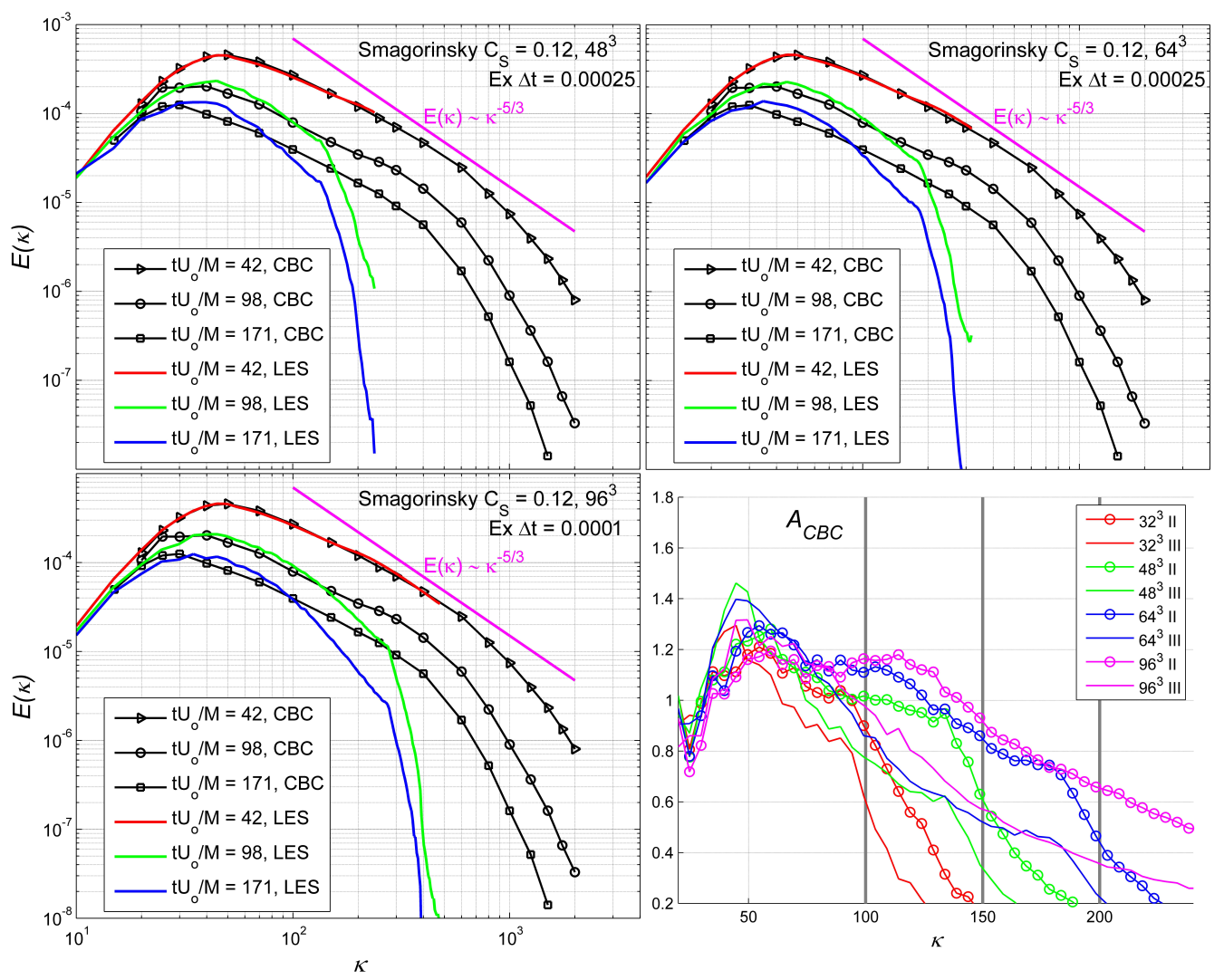

Figure 7. Changes in the energy spectra for $C_{S}=0.12$ with the ECNS on $48^{3}, 64^{3}$ and $96^{3}$ grids. The (bottom-right) plot shows $A_{C B C}$ at different grid resolutions.

One may conclude that beyond $96^{3}$, changes in grid resolution will not alter the spectra for the large scales (up to a wavenumber of 100) because the contribution of $\Delta x$ to $\mathcal{S}(\tau, \Delta x)$ in Equation (11) (or the discretisation error in the SGS model) will be reasonably small. This leads to a more or less constant overall dissipation, governed solely by Smagorinsky model and its constant $C_{S}=0.12$, in this case. Therefore, it is reasonable to assume that for refined grids, one could probably resort to a single value of $C_{S}$, to correctly predict the spectra up the wavenumber at which the spectra seem to dampen. 
Additional tests were conducted using different SGS models and even without one, to find out what causes the excessive dampening at high wavenumbers near the limit of the grid's resolution. Figure 8 shows the results obtained with a pseudo-spectral code with the Germano model. The Germano model was also used in combination with the ECNS but the results obtained were poor, especially near the higher wavenumbers without any changes in the tails. This supported a hypothesis that the tails may arise from the use of a FVM. Therefore, it was deemed sensible to exclude regions that show these damped tails from further analyses on the spectra. The reason behind their occurrence has little relevance to this article but could be worth investigating.

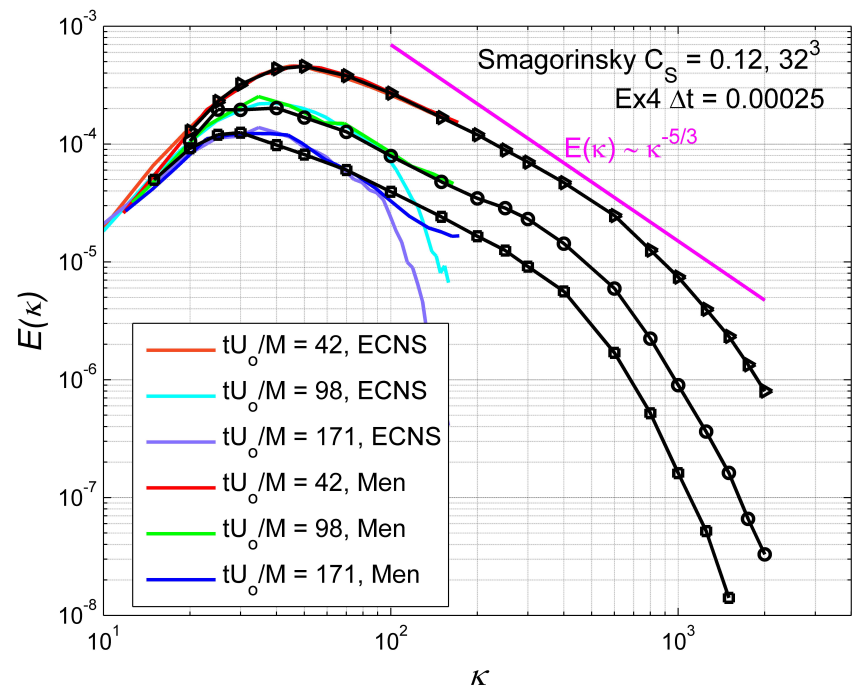

Figure 8. A comparison between the ECNS with the Smagorinsky model and $C_{S}=0.12$ on a $32^{3}$ grid and a pseudo-spectral code with the Germano dynamic model on a $32^{3}$ (Men in the plot) from [26].

Next, an analysis similar to the one followed in [31] is preformed to tune the Smagorinsky model for the ECNS.

\subsection{Tuning the Smagorinsky Model}

To tune the Smagorinsky model in the ECNS, one must first calculate what part of the energy spectra (Stage II and Stage III) can be predicted accurately through the simulations. For example, one may check how closely can the solution on a $64^{3}$ report the spectra up to a wavenumber equal to the cut-off wavenumber on a $32^{3}$ grid and likewise for different resolutions.

To begin with, the Smagorinsky constant is set to $C_{S}=0.12$, on a $32^{3}$ grid. The results are compared with LES data from [26] in Figure 8, which appear to be quite similar, apart from the sudden dampening of energy beyond a wavenumber of $\kappa=100$. Next, the same value of $C_{S}=0.12$ is used (and other values close to 0.12 , for example, $0.11,0.115,0.125$ and 0.13 ) to obtain the energy spectra on grids up to $96^{3}$. In either case, it is ensured that the time integration introduces no numerical dissipation (see Section 5.2).

Next, one sets three limits $\mathcal{A}, \mathcal{B}$ and $\mathcal{C}$, where $\mathcal{A}$ is only a part of the simulated energy spectrum on a $32^{3}$ grid, $\mathcal{B}$ a part of the simulated spectrum on $48^{3}$ and $\mathcal{C}$ for a $64^{3}$ grid. Figure 9 shows these three limits with grey lines. One notices that these limits are not equal to the cut-off wavenumber of the pertinent grid. This however ensures that the damped tail noticed so far with the spectra obtained using the ECNS, are removed from the analysis due to the reasons mentioned earlier.

To estimate the error incurred while predicting the part of the spectrum up to the cut-off wavenumber obtained on a $48^{3}$ grid or $\mathcal{B}$ but using a $80^{3}$ grid, the spectra obtained with the $80^{3}$ grid are integrated till the corresponding grey line that indicates $\mathcal{B}$ in Figure 9 . Then the difference in energy with respect to the experimental solution integrated till the wavenumber corresponding to $\mathcal{B}$ 
is calculated. This is repeated for all logical combinations (an illogical combination would be trying to assess the prediction on $\mathcal{C}$ with a $48^{3}$ grid) and the resulting errors are summarised in percentage within Table 3 (see Figure 9 to interpret results).

Table 3. A summary of errors (reported in percentage) using the energy spectra obtained by CBC [25].

\begin{tabular}{|c|c|c|c|c|c|c|c|c|c|c|c|}
\hline \multirow[b]{2}{*}{$C_{S}$} & \multirow[b]{2}{*}{ Case } & \multicolumn{4}{|c|}{ II (\%) } & \multicolumn{6}{|c|}{ III (\%) } \\
\hline & & 32 & 48 & 64 & 80 & 96 & 32 & 48 & 64 & 80 & 96 \\
\hline \multirow{3}{*}{0.115} & $\mathcal{A}$ & 15 & 19 & 20 & 13.4 & 7.8 & 10.9 & 18.8 & 20.3 & 15.6 & 9.4 \\
\hline & $\mathcal{B}$ & & 12.2 & 14.9 & 12.6 & 8.1 & & 7.5 & 10 & 7.5 & 3.9 \\
\hline & $\mathcal{C}$ & & & 10.8 & 10.1 & 6.5 & & & 3 & 1.1 & 1.1 \\
\hline \multirow{3}{*}{0.12} & $\mathcal{A}$ & 11.2 & 15.5 & 18.1 & 12.9 & 7.8 & 6.3 & 14.1 & 18.8 & 14 & 9.3 \\
\hline & $\mathcal{B}$ & & 8.1 & 12.6 & 10.1 & 6.8 & & 2.5 & 7.5 & 6.3 & 3.8 \\
\hline & $\mathcal{C}$ & & & 7.1 & 7.7 & 4.8 & & & $\approx 0$ & $\approx 0$ & 2.2 \\
\hline \multirow{3}{*}{0.125} & $\mathcal{A}$ & 6.9 & 12.9 & 16.3 & 12 & 6.9 & $\approx 0$ & 10.9 & 15.6 & 14.1 & 9.4 \\
\hline & $\mathcal{B}$ & & 4.1 & 9.5 & 8.8 & 6.1 & & 1.3 & 5 & 3.8 & 2.5 \\
\hline & $\mathcal{C}$ & & & 3.6 & 5.4 & 3 & & & 3.3 & 2.2 & 3.3 \\
\hline \multirow{3}{*}{0.13} & $\mathcal{A}$ & 12.1 & 14.3 & 17.6 & 13 & 7.6 & $\approx 0$ & 14.2 & 18.4 & 15.7 & 9.6 \\
\hline & $\mathcal{B}$ & & 7.7 & 10.1 & 9.9 & 6.3 & & 2.4 & 7.3 & 6.2 & 3.5 \\
\hline & $\mathcal{C}$ & & & 6.8 & 7.9 & 4.6 & & & 3.3 & 2.6 & 3.5 \\
\hline
\end{tabular}

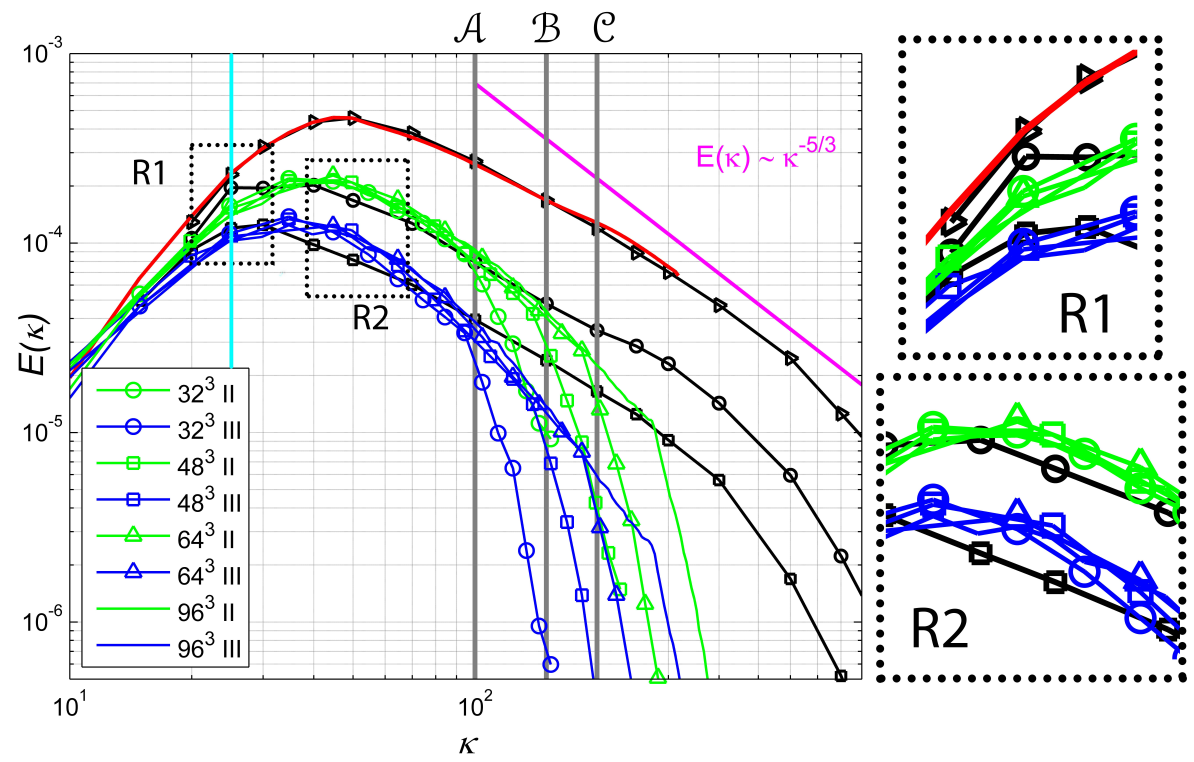

Figure 9. Changes in the energy spectra with grid resolution for ECNS and $C_{S}=0.12$.

It is important to note that the error is calculated as the difference between the experimentally determined (from [25]) and the simulated TKE and not as the root-mean-square error of the differences observed in the spectra at different wavenumbers. This is because the simulated spectra show two regions of marked deviations at smaller wavenumbers (indicated in Figure 9 as $R 1$ and R2), which as it appears, do not change with grid resolution and could lead to a constant offset in the calculated errors. Further, these region are also noticed in Figure 8, when simulated with a pseudo-spectral code and the Germano dynamic model (indicated in the plot with Men).

In general, the errors are lower for Stage III than Stage II. With $C_{S}=0.12$ one notices a negligible error in predicting $\mathcal{C}$ for Stage III, especially with finer grids. But in this case, the corresponding errors for Stage II are higher. On the other hand, $C_{S}=0.125$ leads to a better result for Stage II than Stage III. Increasing the $C_{S}$ beyond 0.125 or reducing it to 0.115 , leads to a higher error. Therefore, one may conclude that an optimal $C_{S}$ belongs to the range [0.12, 0.125], with regards to the test case. Also, as hypothesised through Equation (11), this range of values is quite independent of the grid resolution, 
provided it is enough to resolve the large scales correctly; possibly due the absence of numerical dissipation guaranteed by the ECNS approach (and the low time step).

Another point worth mentioning is the shape of the experimental spectrum at Stage II (see R1 in Figure 9). One notices a sharply placed data point at a wavenumber of $\sim 25$ (indicated with a cyan line), as opposed to the smooth spectra at the other stages and the ones obtained through simulations. It is probable that the deviation of the simulated spectra from this data point, contributes to the error at Stage II to a great extent, given the relatively high energy content at that wavenumber.

\subsubsection{OpenFOAM and the Influence of Numerical Dissipation}

The procedure in Section 5.4 is repeated with OpenFOAM using the spatial discretisation mentioned in Table 1 , to obtain a value of $C_{S}=0.135$ on a $32^{3}$ grid, as shown in Figure 10. It was ensured that the time integration does not introduce numerical dissipation by using a very small time step of $0.0001 \mathrm{~s}$.

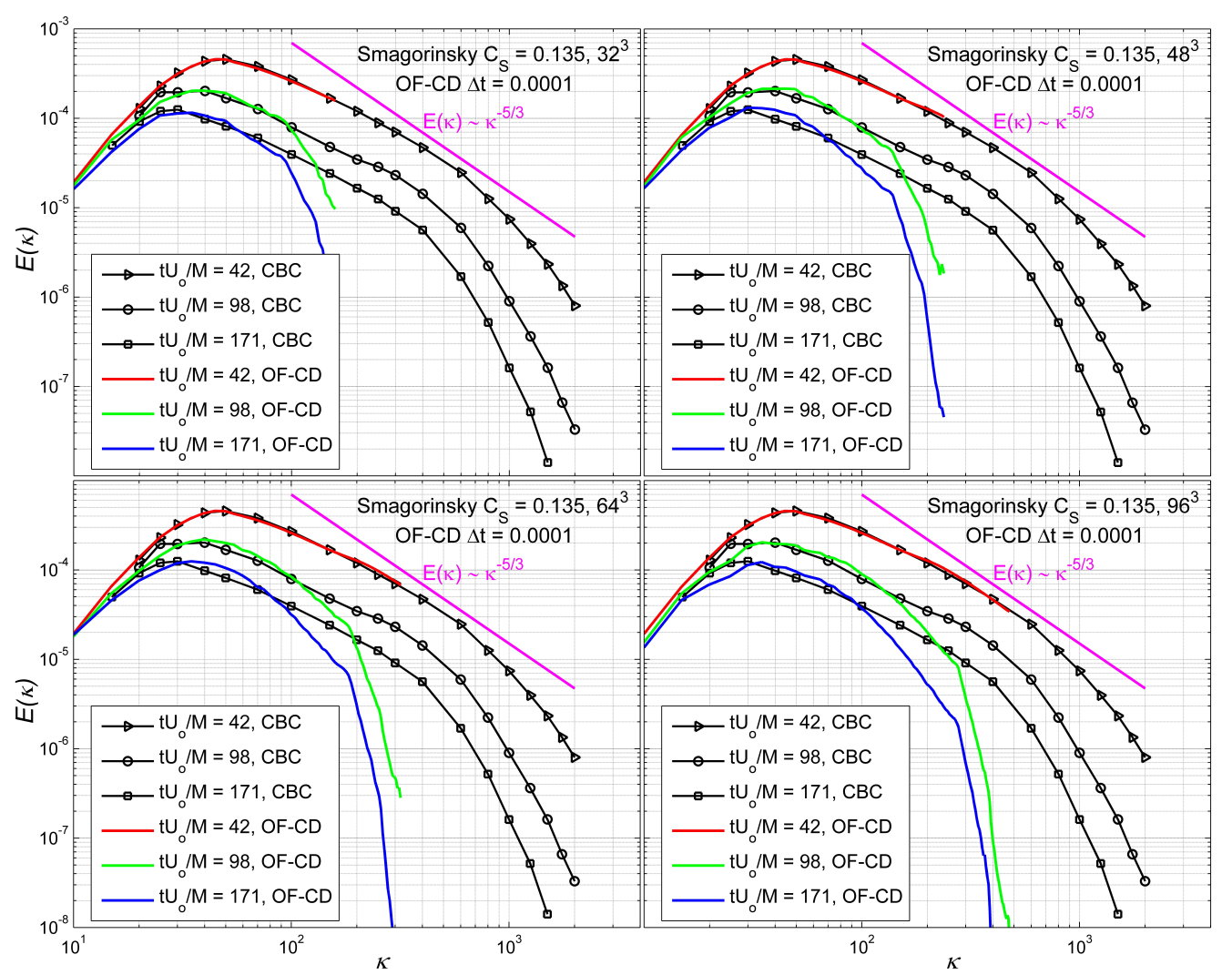

Figure 10. Spectra obtained with the OpenFOAM approach and $C_{S}=0.135$ on $32^{3}, 48^{3}, 64^{3}$ and $96^{3}$ grids (clockwise from (top-left)).

Upon increasing the grid resolution, one notices that the OpenFOAM approach of a central difference scheme on a collocated grid behaves similar to the ECNS approach of an EC scheme on a staggered grid. One also obtains the damped tails that were noticed with the ECNS, indicating that these are possibly inherent to an FVM using the Smagorinsky model. Further, the tails dampen above a certain wavenumber, below which, the scales (the large energy-containing ones) remain nearly unaffected with changes in grid resolution (see Figure 10). Although on a $96^{3}$ grid one does see some dampening of the large scales at Stage III, Stage II of the process remains nearly unaffected.

OpenFOAM is also used with the upwind schemes, QUICK and LU mentioned in Section 4. An immediate consequence is the strong effect of numerical dissipation leading to a damping of the spectra, as shown in Figure 11. As the grid resolution in increased (only shown with QUICK), it is observed that there is a reduction in dissipation as the large scales move towards their experimentally 
established values. However, the spectra as whole does not recover its correct shape indicating incorrect decay rates at multiple wave numbers. However, the 3rd order formulation of QUICK introduces relatively lesser dissipation than its 2nd order counterpart, LU (compare top-left and top-right in Figure 11).

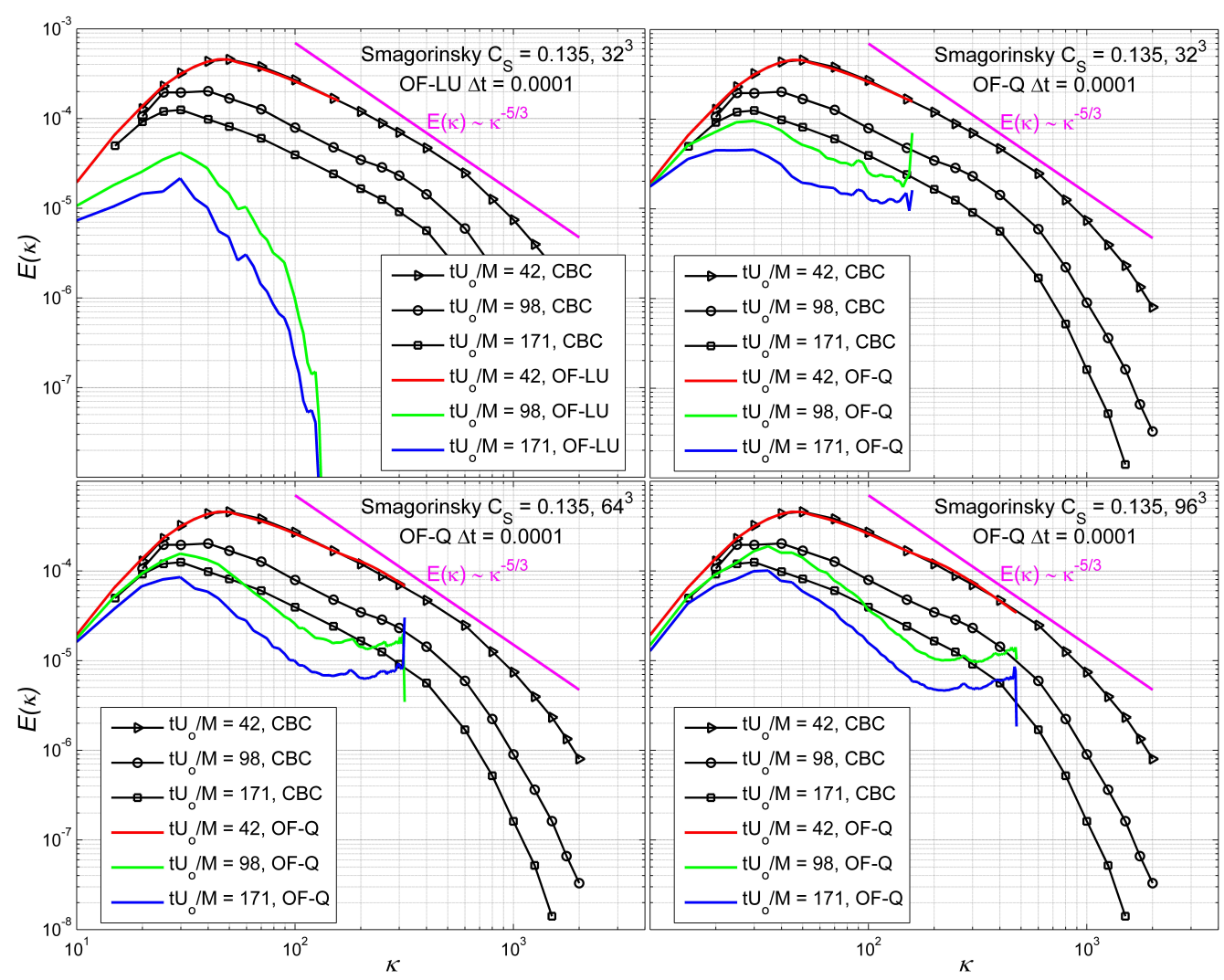

Figure 11. Spectra obtained on collocated grids with two different upwind schemes. (top-Left): LU on a $32^{3}$ grid. (Top-right) and (bottom): QUICK on $32^{3}, 64^{3}$ and $96^{3}$ grids.

In short, one is able to propose a suitable value of $C_{S}$ that allows finer grids to predict a fair regime of the large scales correctly with the ECNS and OpenFOAM. But on the other, one must not forget that the overall error, especially on the coarser grids, is a combination of the contributions from the diffusive and the SGS operators, which can only be studied separately and practically if the Reynolds number is high enough to neglect the diffusive operator.

Upon conducting relevant tests on the LES of the ABL and the wake of a model wind turbine in a wind tunnel; and comparing the results so-obtained against those generated with other LES codes, it was noticed that the values of $C_{S}$ deduced in this article are rather accurate for the simulation of high Reynolds number flows. Details on these tests and the results so obtained can be found in Mehta [28].

Out of the tests conducted, the simulation of the ABL is of much relevance to the wind energy community. LES has evolved as an important tool for the simulation of wind farm aerodynamics to study phenomena such as the effect of large wind farms on local weather, the meandering of a turbine's wake, wind power generation, to name a few [32]. In either case, one requires an accurate simulation of the ABL to create a suitable velocity field which encompasses the turbulent conditions that are present in the ABL. Therefore, after obtaining a possibly appropriate value of $C_{S}$ for the ECNS, it was first assessed if the value was suitable in terms of simulating the correct velocity profile and turbulence spectra within a neutral ABL. The results from this test will be described briefly in the next section. 


\section{Simulation of the ABL with the ECNS}

To standardise the ABL simulations, the domain is chosen to match the one described by Porté-Agel et al. [33] in the study of neutral ABLs, the dimensions of which are listed in Appendix E. Further, Appendix E contains important details on the velocity profile expected inside the simulated neutral ABL (log-law profile) and the simulation set-up. The results discussed in this section are obtained with the ECNS in combination with the Smagorinsky model to perform an LES.

It is important to note that the values for $C_{S}$ derived earlier, were done so for isotropic flows. However, the flow within an ABL is anisotropic near the surface, which in turn will affect the dissipation rate. Mason [34] proposed the following ad hoc function to reduce the value of the Smagorinsky constant near the surface in keeping with increased anisotropy and loss of scale invariance [33].

$$
C_{S_{y}}=\left[C_{S_{o}}^{-n}+\left\{\kappa+\left(\frac{y}{\Delta}+\frac{y_{0}}{\Delta}\right)\right\}^{-n}\right]^{-\frac{1}{n}},
$$

where $C_{S_{y}}$ is the Smagorinsky constant at height $y$ above the ground, $C_{S_{o}}$ is the Smagorinsky constant for homogeneous isotropic turbulence, $\Delta$ is the overall grid resolution and $n$ is an integer of choice.

To tune the ECNS for ABL flows, the values of $C_{S}$ found earlier were used in combination with a value of 1 or 2 for $n$. The results obtained were analysed in terms of the average streamwise velocity profile that should be close to the log-law for the ABL (see Appendix E) and the energy spectra as a function of height. For clarity, only the results relevant to this article are discussed here; more details can be found in Mehta [28]. The combinations of $C_{S}$ and $n$ discussed in this section are $C_{S}=0.125$, $n=1$ and $C_{S}=0.12, n=2$, which rendered the most accurate velocity profiles.

The tuning was performed on $64^{3}$ and $96^{3}$ grids. Figure 12 depicts how the velocity prediction improves with resolution, especially in the lower part of the ABL close to the ground, regardless of the tuning parameters. Outside of this region, above $y / L_{y}=0.1$, the tuning parameters do not affect the profile. On a $96^{3}$ grid, the combination $C_{S}=0.125, n=1$, approximates the log-law closely till around $20 \mathrm{~m}$ above the ground, followed by a under estimated velocity regime. On the other hand, the combination $C_{S}=0.120, n=2$, underestimates the velocity below $y / L_{y}=0.1$. Although increasing the grid resolution improves the prediction with both $C_{S}=0.120, n=2$ and $C_{S}=0.125, n=1$, the latter renders a more accurate average velocity profile.

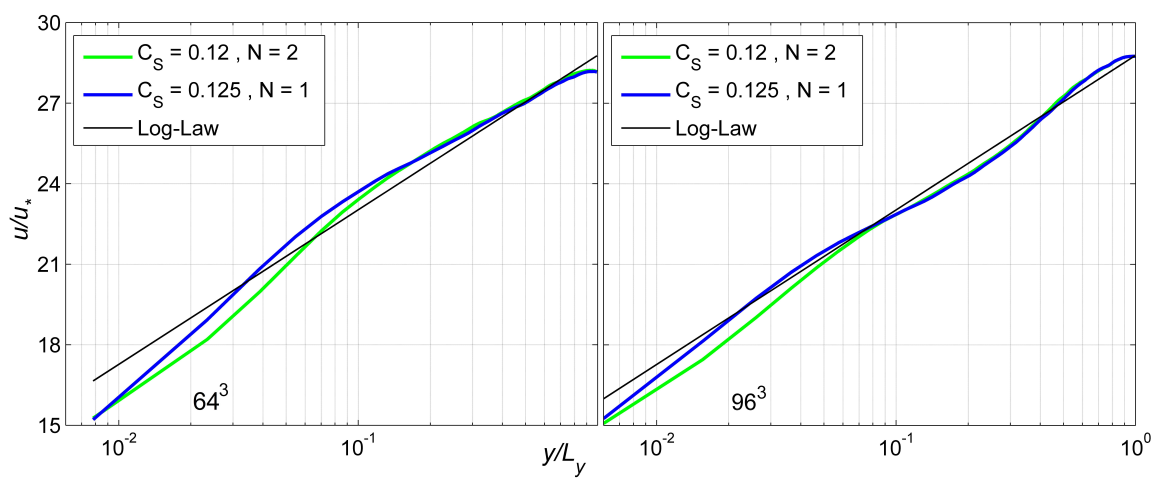

Figure 12. The average streamwise velocity with height for different grids and combinations of the Smagorinsky-Mason model. The $y$-axis is the ratio of the streamwise velocity to the friction velocity, $u_{\star}$ and $x$-axis is the ratio of the height and the height of the domain, $L_{y}$.

For better insight into the nature of turbulence within the simulated ABL, one must look at the spectra at various heights. One expects the spectra to segregate into two regimes. The first with a slope of nearly -1 in the region near the ground and the second with a slope of $-5 / 3$, located away from the ground, as noticed by various studies (see Porté-Agel et al. [33] and references therein). Figure 13 
shows that around $400 \mathrm{~m}$ above the ground, the spectra collapse onto the standard $-5 / 3$ slope for isotropic homogeneous turbulence, more or less, independently of the tuning parameters.

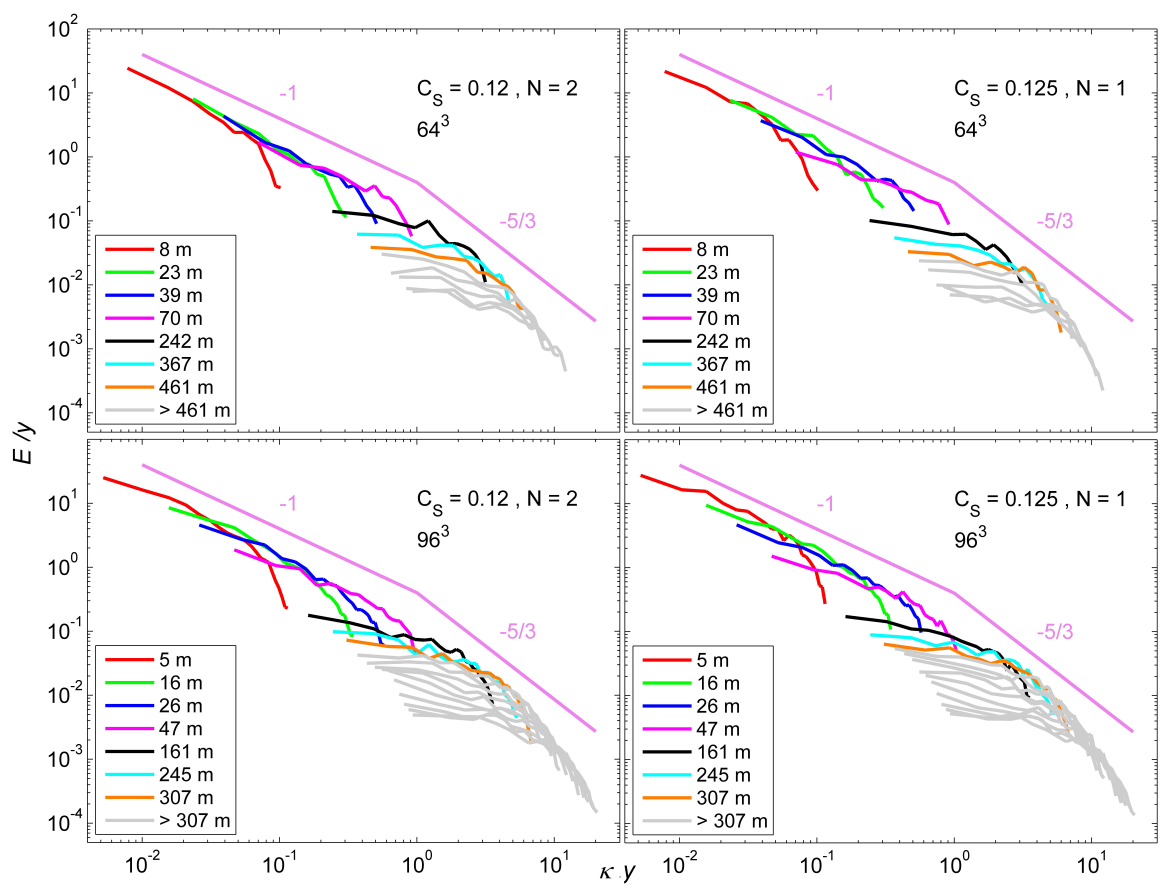

Figure 13. The energy spectra with height on $64^{3}$ (top) and $96^{3}$ (bottom), for $C_{S}=0.12, n=2$ (left) and $C_{S}=0.125, n=1$ (right).

Further, the inertial range clearly exhibits a slope of -1 followed by the sharp dampening of the spectra at higher wavenumbers that was also noticed in the CBC simulations. This can once again be attributed to the FVM used in the ECNS. Therefore, in this case too, one must only take the spectra up to this sharp tail into account.

Figure 13 depicts that the spectra near the ground do not depend much on the combination of the $C_{S}$ and $n$. In fact, they collapse well onto the -1 slope, apart from the sharp dampening at higher wavenumbers. Thus, with nearly similar spectra for either combination, $C_{S}=0.12, n=2$ and $C_{S}=0.125, n=1$, the latter is chosen as it renders a more accurate velocity profile.

\section{Conclusions}

With regards to an implicit EC time integration scheme, one may conclude that it is capable of averting numerical dissipation at large time steps, while remaining stable and ultimately leading to an accurate solution. But in terms of the test case considered in this article, it appears that a non-EC explicit scheme is computationally efficient with small time steps, as long as it is of the same order as the implicit EC scheme (4th order in this case). Using a 2nd order explicit non-EC scheme may not be favourable as even an implicit EC 2nd order scheme requires a reasonably small time step for accuracy.

Further, it is fair to mention that the implicit methods may retain all their advantages in simulations that involve local grid refinement and the presence of walls, which may require a non-uniform grid to obtain accurate results. In such cases, the stability criteria may be very strict for non-EC explicit methods to resolve the refined regions, possibly rendering them unworkable. On the other hand, most LES studies on wind farm aerodynamics mentioned in literature, prefer to model the shear stress at the surface instead of refining the flow near it to reduce computational requirements, thus favouring the non-EC explicit methods. To harness the accuracy and stability of implicit-EC methods, a detailed study of the procedure used to solve their system of equations must be performed to assess if the computational time for implicit-EC methods could be reduced. With regards to spatial discretisation, it is noticed that the OpenFOAM approach leads to large scale behaviour that 
is similar to what one notices with the ECNS approach. No pronounced advantages of latter were found, which would make it more favourable for applications such as wind farm aerodynamics or atmospheric simulations. Further, the damped tail in the energy spectra, noticed with either approach can be attributed with confidence to the use of an FVM with the Smagorinsky model. This simple model does not account for changes in dissipation with scale, leading to excessive damping at smaller scales (as confirmed with two different codes). With either code, one obtains a value of the Smagorinsky constant that is nearly independent of the grid resolution (within reasonable bounds), as long as the numerical schemes are dissipation-free.

Following a series of tests, of which the simulation of a neutral ABL is presented here, one may conclude that an EC scheme on a staggered grid and the central difference scheme on a collocated grid could be similar in the simulation of high Reynolds number flows (on uniform grids) with the simple Smagorinsky model, which is the motivation behind developing the ECNS.

Author Contributions: D.M. and Y.Z. are doctoral students and also the corresponding authors, who have contributed equally to this article; A.v.Z. and H.B. supervised the doctoral research carried out by D.M. and Y.Z.

Funding: This article was prepared under the aegis of the $\mathrm{PhD}$ research organised by the Energy research Centre of the Netherlands (ECN, now TNO) as part of the Marie Curie Initial Training Networks's project MARE-WINT (new MAterials and REliability in offshore WINd Turbines technology). MARE-WINT was directly funded by the European Commission as part of the Seventh Framework Programme under the Grant Agreement numbered 309395. MARE-WINT's contribution is much acknowledged.

Acknowledgments: The authors would like to thank ECN (now TNO) for supplying their Energy-Conserving Navier-Stokes (ECNS) code to carry out the research mentioned in this article.

Conflicts of Interest: The authors declare no conflict of interest.

\section{Abbreviations}

The following abbreviations are used in this manuscript:

$\begin{array}{ll}\text { LES } & \text { Large eddy simulation } \\ \text { EC } & \text { Energy-conserving } \\ \text { DNS } & \text { Direct numerical simulation } \\ \text { SGS } & \text { Subgrid scale } \\ \text { FVM } & \text { Finite volume method } \\ \text { NS } & \text { Navier-Stokes } \\ \text { KE } & \text { Kinetic energy } \\ \text { TKE } & \text { Turbulence kinetic energy } \\ \text { ABL } & \text { Atmospheric boundary layer } \\ \text { OpenFOAM } & \text { Open source field operation and manipulation } \\ \text { ENCS } & \text { Energy-conserving Navier-Stokes } \\ \text { ECN } & \text { Energy research Centre of the Netherlands } \\ \text { TNO } & \text { Nederlandse Organisatie voor toegepast-natuurwetenschappelijk onderzoek } \\ \text { Ex4 } & \text { 4th order explicit Runge-Kutta scheme } \\ \text { Im2 } & \text { 2nd order implicit EC scheme } \\ \text { Im4 } & \text { 4th order implicit EC scheme } \\ \text { QUICK } & \text { Quadratic upwind interpolation for convective kinematics } \\ \text { LU } & \text { Linear upwind } \\ \text { CBC } & \text { Comté-Bellot and Corrsin } \\ \text { CFL } & \text { Courant Friedrichs Lewy } \\ \text { GMRES } & \text { Generalised minimal residual method } \\ \text { PISO } & \text { Pressure-implicit with splitting of operators } \\ & \end{array}$

\section{Appendix A. Details on the Codes and Numerical Schemes}

This appendix describes the various numerical schemes mentioned in Section 4 and provides some information about pressure-velocity coupling within the ECNS and OpenFOAM. 
Appendix A.1. ECNS

The Ex4 is the classical Runge-Kutta method that is explicit and quick but restricted by stability at very large time steps. The continuity equation is solved with the velocity field decoupled from the pressure field. Apart from the Ex4, various lower-order explicit methods have been used for the LES of wind farm aerodynamics with small time steps for accuracy and stability. This was the motivation behind proposing the implicit EC methods, which are not only accurate but also stable at very large time steps.

The Im 2 is a one-stage method based on the Gauss quadrature, whereas the Im 4 is a two-stage method. Both are solved as a coupled linearised system (pressure and velocity field together) obtained using Newton's method and requiring data from the previous time levels. This is also known as the full Newton method and requires an exact Jacobian to solve the linear system at every iteration towards the solution of a given time step. This ensures that the time scheme remains EC and unconditionally stable, which are the benefits that EC time schemes offer.

\section{Appendix A.2. Pressure-Velocity Coupling}

Improper coupling of pressure and velocity within a solver can spuriously affect the dissipation of KE. However, this is not the case with the ECNS as the use of a staggered grid ensures perfect pressure-velocity coupling [7]. On the other hand, the pressure-implicit with splitting of operators (PISO) algorithm was used within OpenFOAM. PISO does not resolve the pressure-velocity coupling completely but approximates it with predictor-corrector steps. In such a case, one expects the pressure to incorrectly contribute to the change in KE (numerical dissipation) if the pressure-velocity system is not iterated to machine precision. However, the direct effect of using PISO on the results was carefully assessed, following which, a good number of predictor-corrector steps were taken to achieve a tolerance that does not affect the KE spuriously. A tolerance of $10^{-10}$ and 2 predictor-corrector steps are sufficient. Further changes did not alter the results noticeably.

\section{Appendix B. Other Test Cases Simulated and Considered}

Ref. [35] conducted an experiment similar to CBC but at a higher Reynolds number around 121,000 , the spectra from which show an extended inertial range with a clear $-5 / 3$ slope. Unlike the experiment by [25], the mean velocity along the wind tunnel in [35]'s case was not constant. One only knows the locations along the wind tunnel where the data was gathered but to simulate the same, one also requires the time taken by the flow to traverse these locations. One can certainly approximate the time but not without introducing an error into an already complex system of errors. Thus, the CBC experiment for this article. Nevertheless, [35]'s case was also simulated and the $-5 / 3$ slope in the spectra was obtained regardless of the time instances at which the data was gathered.

Another test case that is of relevance to this article, is the decay of the Taylor Green vortex [36-38]. It is the unsteady decay of a three dimensional (or two dimensional) velocity field specified by initial conditions defined by Taylor and Green [39]. The time-varying flow field that results from the decay can be obtained exactly for a two dimensional initial field; reference solutions obtained using DNS are available for a three dimensional initial field.

Although this case was used for the initial verification of ECNS at low Reynolds numbers [40], its use for Reynolds numbers of 30,000 or higher was not feasible due to the requirement of a DNS solution for verification. One could still use LES to simulate the decay of a three dimensional flow field and assess whether the $-5 / 3$ slope of the spectra is obtained or not. However, we decided to choose the $\mathrm{CBC}$ test case solely due to the experimental data available (as an alternative to DNS, which is computationally infeasible for a Reynolds number of 30,000). 


\section{Appendix C. Generation of the Initial Velocity Field for the СВС Case}

To begin with, the energy spectrum at Stage I is used to synthesise a random velocity field that bears the experimentally reported TKE and variances in turbulent fluctuations within a suitable domain. To ensure that the periodic boundaries used in the simulation do not lead to spurious correlations, the size of the domain in either direction must be large as compared to the longitudinal and transverse length scales of the flow. The domain is chosen to be $L_{b o x}=10.8 M_{g} \sim 55 \mathrm{~cm}$ in size, based on previous LES studies [26,41]. The velocity field is generated spectrally using Rogallo's algorithm and then converted into discretised physical space through an inverse-Fourier transformation [42]. The algorithm distributes the TKE of the flow across the range of Fourier modes in compliance with the energy spectrum provided as input, which in this case comes from the experimental data. During the conversion, it was noticed that the energy of the highest wavenumber modes (the smallest scales of the velocity field) is reduced in physical space. Although Parseval's theorem holds but inaccuracies in the fast-Fourier transform may have led to this dampening. This is rescaled to the spectrum at Stage I by multiplying the Fourier modes individually with the factor that equals the damping they incurred upon conversion to physical space.

Although one generates the velocity field in Fourier space while ensuring that it is divergence free; upon projecting the Fourier field on a discrete Cartesian grid in physical space, one may obtain a field that is not divergence free, at least in terms of how the divergence is defined in the FVM-based code. As a result, it is important to adapt the velocity field to an NS solver to adjust its divergence and establish space-time correlations within the flow-field as they would exist in reality [5]. To do so, the field is left to decay in accordance with the NS equations and the Smagorinsky model for a while. After its TKE has reduced significantly, the field is scaled up in Fourier space to the energy spectrum at Stage I, similar to what is done to correct the damping of the smallest scales due to inverse Fourier transforms, i.e., by scaling the energy at individual wavenumbers till it matches its counterpart at Stage I.

\section{Appendix D. Computational Time and System of Equations for Implicit Schemes}

The extra computational requirements for $\operatorname{Im} 2$ and $\operatorname{Im} 4$ arise from the methods used to solve the system of equations within these time integration scheme, which are first linearised using the Newton method. The linearisation that follows, reaches its limit of accuracy below a time step of $0.00025 \mathrm{~s}$ as shown in Figure 4. One notices in Figure 4 that at higher resolutions, the error levels off at a higher magnitude although the machine precision should remain the same. This is because one may require more number of Newton iterations at a higher resolution to reduce the iterative error in solving the implicit schemes. This occurs because the non-linear residual needed for accuracy is very low $\left(10^{-15}\right)$ or is not reached after the prescribed number of linearisation iterations. Further, it is also important to ensure that the error that results from the linearisation of the system of equations, is reduced to a value much smaller than the overall discretisation error at every time step. An error from linearisation that is comparable to the overall discretisation error can reduce the accuracy of the implicit methods considered here. Although this article does not present the results, it was noted that the accuracy of the Im 4 is very strongly related to the tolerance set for the linearisation. For an overall accuracy of $10^{-4}$, one must ensure a non-linear residual of $10^{-8}$ or lower. If one desires an overall error of $10^{-6}$, the linearisation must be done till a tolerance of $10^{-10}$; and so on for smaller time steps. Not doing so can even render the Im4 less accurate than the Ex4.

Further, it was also assessed whether the computational time is a result of extra linearisation iterations being done even after reaching the tolerance. This was not the case and most time steps were resolved with 4 to 6 linearisation iterations. Also within each linearisation step, the generalised minimal residual method (GMRES) was used to solve the linearised system to obtain the pressure and velocity fields. Overall, the process for each time advancement was directly connected to the linearisation iterations needed to a reach a tolerance that guarantees accuracy and the minimisation of numerical dissipation, with each linearisation iteration requiring a GMRES solve. 
Finally, a tolerance comparable to that of the linearisation was generally reached within 200 iterations which comprised the computationally intensive part of the Im4 solver (the simulations were aimed at $10^{-12}$ for lower values of linearisation tolerance such as $10^{-10}$ ). Once again, it was checked that once the expected tolerance was reached, the GMRES was stopped.

\section{Appendix E. ABL Simulation}

This section contains details on the log-law velocity profile and the case set-up for simulating the neutral ABL.

\section{Appendix E.1. Log-Law Profile}

A mathematical representation of the velocity in the ABL that is commonplace, is the log wind profile [43] or,

$$
u(y)=\frac{u_{\star}}{\kappa} \ln \left(\frac{y}{y_{0}}\right)+\psi_{M},
$$

where $u$ is the streamwise velocity parallel to the ground, $y$ is the height above the ground, $u_{\star}$ is the friction velocity, $\kappa$ is the von Kármán constant equalling $0.4, y_{0}$ is the aerodynamic roughness length and $\psi_{M}$ is stability correction, which in the case of a neutral ABL is 0 .

The aim is to use LES and generate a velocity profile determined by Equation (A1) for a given surface roughness and the desired wind velocity at a certain height, commonly chosen as the inflow velocity that a wind turbine should have at hub-height when placed within the simulated ABL $[31,44]$. The roughness length on the other hand, must be changed as per the terrain.

\section{Appendix E.2. Simulation Set-Up}

To standardise the simulations with ECNS, the domain is designed to be the same as that used often by Porté-Agel et al. [33] in the study of neutral ABLs, the dimensions of which are as follows: height $L_{y}=1000 \mathrm{~m}$, width and length $L_{x}=L_{z}=2 \pi L_{y} \mathrm{~m}$. Further, the density of air is set to $\rho=1.225 \mathrm{kgm}^{-3}$, whereas the molecular viscosity is $18.1 \mu$ Pas. For the case considered in this article, the roughness length is $y_{0}=0.01 \mathrm{~m}$ and the friction velocity is $u_{\star}=0.3561 \mathrm{~ms}^{-1}$.

All simulations are run with a time step of $\Delta t=0.125 \mathrm{~s}$ to ensure the absence of numerical dissipation, the maximum Courant number permitted with the Ex4 is 0.01 , which is conservative as per the observations from the $\mathrm{CBC}$ simulations. The ABL simulations done till the flow statistics reach a quasi-steady state, i.e., they do not change as averages over a fixed period of time (10 min averaged data in this case). This generally required simulating a few hours of real time, following which, flow quantities averaged over 10 minutes showed little change with time.

\section{References}

1. Ghosal, S. An analysis of numerical errors in large-eddy simulations of turbulence. J. Comput. Phys. 1996, 125, 187-206. [CrossRef]

2. Vreman, B.; Geurts, B.; Kuerten, H. Comparison of numerical schemes in large-eddy simulation of the temporal mixing layer. Int. J. Numer. Methods Fluids 1996, 22, 297-311. [CrossRef]

3. Meyers, J.; Geurts, B.J.; Baelmans, M. Database analysis of errors in large-eddy simulation. Phys. Fluids 2003, 15, 2740-2755. [CrossRef]

4. Meyers, J.; Geurts, B.J.; Sagaut, P. A computational error-assessment of central finite-volume discretizations in large-eddy simulation using a Smagorinsky model. J. Comput. Phys. 2007, 227, 156-173. [CrossRef]

5. Viré, A.; Knaepen, B. On discretization errors and subgrid scale model implementations in large eddy simulations. J. Comput. Phys. 2009, 228, 8203-8213. [CrossRef]

6. Smagorinsky, J. General circulation experiments with the primitive equations, Part 1: The basic experiment. Mon. Weather Rev. 1963, 91, 99-164. [CrossRef]

7. Verstappen, R.W.C.P.; Veldman, A.E.P. Symmetry-preserving discretization of turbulent flow. J. Comput. Phys. 2003, 187, 343-368. [CrossRef] 
8. OpenFOAM. The Open Source CFD Toolbox, Version 2.2.2; OpenCFD Limited (ESI Group): Bracknell, UK, 2017.

9. Sanderse, B. ECNS: Energy-Conserving Navier-Stokes Solver: Verification for Steady Laminar Flows; Technical Report ECN-E-11-042; Energy Research Centre of The Netherlands: Sint Maartensvlotbrug, The Netherlands, 2011.

10. Pope, S.B. Turbulent Flows; Cambridge University Press: Cambridge, UK, 2000.

11. Sagaut, P. Large Eddy Simulation for Incompressible Flows, 3rd ed.; Springer-Verlag: Berlin/Heidelberg, Germany, 2006.

12. Leonard, A. Energy Cascade in Large-Eddy Simulations of Turbulent Fluid Flows. Adv. Geophys. 1974, 18, 237-248.

13. Lesieur, M.; Metais, O. New Trends in Large-Eddy Simulations of Turbulence. Annu. Rev. Fluid Mech. 1996, 28, 45-82. [CrossRef]

14. Meneveau, C.; Katz, J. Scale-Invariance and Turbulence Models for Large-Eddy Simulation. Annu. Rev. Fluid Mech. 2000, 32, 1-32. [CrossRef]

15. Meyers, J.; Sagaut, P. On the model coefficients for the standard and the variational multi-scale Smagorinsky model. J. Fluid Mech. 2006, 569, 287-319. [CrossRef]

16. Lilly, D.K. The representation of small-scale turbulence in numerical simulation experiments. NCAR Manuscr. 1966. [CrossRef]

17. Bechmann, A. Large-Eddy Simulation of Atmospheric Flow over Complex Terrain. Ph.D. Thesis, Risø National Laboratory-Technical University of Denmark, Roskilde, Denmark, 2006.

18. Sørensen, N.N. General Purpose Flow Solver Applied to Flow over Hills. Ph.D. Thesis, Risø National Laboratory, Roskilde, Denmark, 1995.

19. Michelsen, J.A. Basis3d-A Platform for Development of Multiblock PDE Solvers; Technical Report AFM 92-06; Technical University of Denmark: Kongens Lyngby, Denmark, 1992.

20. Meyers, J.; Geurts, B.J.; Baelmans, M. Optimality of the dynamic procedure for large-eddy simulations. Phys. Fluids 2005, 17, 045108. [CrossRef]

21. Sanderse, B. Energy-Conserving Discretization Methods for the Incompressible Navier-Stokes Equations. Ph.D. Thesis, Eindhoven University of Technology, Eindhoven, UK, 2013.

22. Grindstein, F.F.; Margolin, L.G.; Rider, W.J. Implicit Large Eddy Simulation: Computing Turbulent Fluid Dynamics, 1st ed.; Cambridge University Press: Cambridge, UK, 2007.

23. Meyers, J.; Meneveau, C.; Geurts, B.J. Error-landscape-based multiobjective calibration of the Smagorinsky eddy-viscosity using high-Reynolds-number decaying turbulence data. Phys. Fluids 2010, 22, 125106. [CrossRef]

24. Perić, M.; Ferziger, J. Computational Methods for Fluid Dynamics, 3rd ed.; Spring-Verlag: Berlin/Heidelberg, Germany, 2002.

25. Comte-Bellot, G.; Corrsin, S. Simple Eulerian time correlation of full-and narrow-band velocity signals in grid-generated, 'isotropic' turbulence. J. Fluid Mech. 1971, 48, 273-337. [CrossRef]

26. Meneveau, C.; Lund, T.S.; Cabot, W.H. A Lagrangian dynamic subgrid-scale model of turbulence. J. Fluid Mech. 1996, 319, 353-385. [CrossRef]

27. Frisch, U. Turbulence, the Legacy of A.N. Kolmogorov; Cambridge University Press: Cambridge, UK, 1995.

28. Mehta, D. To Conserve, or Not to Conserve... Ph.D. Thesis, Delft University of Technology, Delft, The Netherlands, 2016.

29. Lignarolo, L.; Mehta, D.; Stevens, R.; Yilmaz, A.; Meyers, J.; Andersen, S.; van Kuik, G.; Meneveau, C.; Holierhoek, J.; Simão-Ferreira, C.; et al. Validation of four LES and a vortex model against PIV measurements of the near wake of an actuator disk and a wind turbine. Renew. Energy 2015, 111, 510-523..

30. Deardorff, J.W. A numerical study of three-dimensional turbulent channel flow at large Reynolds numbers. J. Fluid Mech. 1970, 41, 453-480. [CrossRef]

31. Meyers, J. Error-Landscape Assessment of Large-Eddy Simulations: A Review of the Methodology. J. Sci. Comput. 2011, 49, 65-77. [CrossRef]

32. Mehta, D.; van Zuijlen, A.; Koren, B.; Holierhoek, J.; Bijl, H. Large Eddy Simulation of wind farm aerodynamics: A review. J. Wind Eng. Ind. Aerod. 2014, 133, 1-17. [CrossRef]

33. Porté-Agel, F.; Meneveau, C.; Parlange, M.B. A scale-dependent dynamic model for large-eddy simulation: Application to a neutral atmospheric boundary layer. J. Fluid Mech. 2000, 415, 261-284. [CrossRef] 
34. Mason, P.J. Large-eddy simulation of the convective atmospheric boundary layer. J. Atmos. Sci. 1989, 46, 1492-1516. [CrossRef]

35. Kang, H.; Chester, S.; Meneveau, C. Decaying turbulence in an active-grid-generated flow and comparisons with large-eddy simulation. J. Fluid Mech. 2003, 480, 129-160. [CrossRef]

36. Fauconnier, D.; de Langhe, C.; Dick, E. Construction of explicit and implicit dynamic finite difference schemes and application to the large-eddy simulation of the Taylor-Green vortex. J. Comput. Phys. 2009, 228, 8053-8084. [CrossRef]

37. Aubard, G.; Stefanin Volpiani, P.; Gloerfelt, X.; Robinet, J. Comparison of subgrid-scale viscosity models and selective filtering strategy for large-eddy simulations. Flow Turbul. Combust. 2013, 91, 497-518. [CrossRef]

38. El Rafei, M.; Könözsy, L.; Rana, Z. Investigation of numerical dissipation in classical and implicit large eddy simulations. Aerospace 2017, 4, 59. [CrossRef]

39. Taylor, G.I.; Green, A.E. Mechanism of the production of small eddies from large ones. Proc. R. Soc. Lon. Ser. A 1937, 158, 499-521.

40. Mehta, D.; van Zuijlen, A.; Bijl, H. Energy-conserving schemes for wind farm aerodynamics. J. Phys. Conf. Ser. 2014, 524, 012157. [CrossRef]

41. Ghosal, S.; Lund, T.S.; Moin, P.; Akselvoll, K. A dynamic localization model for large-eddy simulation of turbulent flows. J. Fluid Mech. 1995, 286, 229-255. [CrossRef]

42. Rogallo, R.S. Numerical Experiments in Homogeneous Turbulence; Technical Report NASA-TM-81315; NASA: Washington, DC, USA, 1981.

43. Panofsky, H.; Dutton, J. Atmospheric Turbulence: Models and Methods for Engineering Applications; John Wiley \& Sons: New York, NY, USA, 1984.

44. Lu, H.; Porté-Agel, F. A modulated gradient model for Large-Eddy Simulation: Application to a neutral atmospheric boundary layer. Phys. Fluids 2010, 22, 1-12. [CrossRef]

(C) 2018 by the authors. Licensee MDPI, Basel, Switzerland. This article is an open access article distributed under the terms and conditions of the Creative Commons Attribution (CC BY) license (http:/ / creativecommons.org/licenses/by/4.0/). 